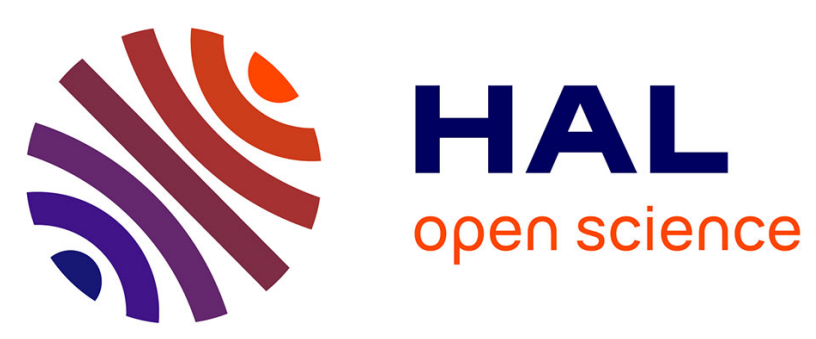

\title{
Laboratory monitoring of CO2 injection in saturated silica and carbonate sands using spectral induced polarization
}

Thomas Kremer, Myriam Schmutz, Alexis Maineult, Pierre Agrinier

\section{To cite this version:}

Thomas Kremer, Myriam Schmutz, Alexis Maineult, Pierre Agrinier. Laboratory monitoring of CO2 injection in saturated silica and carbonate sands using spectral induced polarization . Geophysical Journal International, 2016, 207, pp.1258-1272. 10.1093/gji/ggw333 . insu-01460070

\section{HAL Id: insu-01460070 \\ https://hal-insu.archives-ouvertes.fr/insu-01460070}

Submitted on 7 Feb 2017

HAL is a multi-disciplinary open access archive for the deposit and dissemination of scientific research documents, whether they are published or not. The documents may come from teaching and research institutions in France or abroad, or from public or private research centers.
L'archive ouverte pluridisciplinaire HAL, est destinée au dépôt et à la diffusion de documents scientifiques de niveau recherche, publiés ou non, émanant des établissements d'enseignement et de recherche français ou étrangers, des laboratoires publics ou privés. 


\title{
Laboratory monitoring of $\mathrm{CO}_{2}$ injection in saturated silica and carbonate sands using spectral induced polarization
}

\author{
Thomas Kremer, ${ }^{1,2}$ Myriam Schmutz, ${ }^{3}$ Alexis Maineult ${ }^{1,2}$ and Pierre Agrinier ${ }^{1,2}$ \\ ${ }^{1}$ Institut de Physique du Globe de Paris, Sorbonne Paris Cité, Université Paris Diderot, F-75005 Paris, France. E-mail: alexis.maineult@upmc.fr \\ ${ }^{2}$ Centre de Recherches sur le Stockage Géologique du CO 2 (IPGP/TOTAL/SCHLUMBERGER/ADEME), F-75005 Paris, France \\ ${ }^{3}$ EA4592, Bordeaux INP, ENSEGID, F-33607 Pessac, France
}

Accepted 2016 September 2. Received 2016 September 2; in original form 2015 December 21

\begin{abstract}
SUMMAR Y
Series of experiments were performed to study the spectral induced polarization (SIP) response of sands fully saturated with water, into which gaseous $\mathrm{CO}_{2}$ or $\mathrm{N}_{2}$ was injected, in the frequency range $0.5 \mathrm{~Hz}-20 \mathrm{kHz}$. Three main observations were made. (1) SIP parameters were always most affected by gas injection when the frequency of the injected signal was in the intermediate range $(1<f<20 \mathrm{kHz})$. This point emphasizes the interest of broadening the frequency range of SIP surveys toward intermediate frequencies. It also implies that more work is needed in order to understand and quantify the parasitic effects that occur at this frequency range (EM coupling and electrode polarization). (2) Through all the experiments performed, we were able to distinguish the parameters variations caused by a reduction of the water saturation level (invasion of a resistive gas phase in the porous space) from those resulting from dissolution processes (increase of the electrical conductivity of the saturating water). (3) The quadrature conductivity $\sigma^{\prime \prime}$, which is mainly linked to the capacitive properties and inductive response of the media, always shows relative variations stronger than the resistivity of the media, hence demonstrating the interest of the additional information provided by the SIP method as against the classical resistivity method.
\end{abstract}

Key words: Electrical properties; Hydrogeophysics; Permeability and porosity.

\section{INTRODUCTION}

Carbon capture and storage (CCS), which consists of storing carbon dioxide $\left(\mathrm{CO}_{2}\right)$ in deep, structurally adapted geological formations, is considered today as a viable strategy to reduce the increase of anthropogenic $\mathrm{CO}_{2}$ emissions in the atmosphere. To ensure the long-term efficiency of CCS and prevent environmental impacts associated with $\mathrm{CO}_{2}$ leakage into nearby aquifers, it is imperative for the technical and scientific community to develop and display on-site monitoring tools that allow the detection and quantification of any $\mathrm{CO}_{2}$ transfer out of the targeted geological formation.

Several approaches are possible (e.g. Liu 2012). Geochemical analysis can be applied to monitor the presence of dissolved species that could result from $\mathrm{CO}_{2}$ dissolution in water and/or its consequent acidification (e.g. Carroll et al. 2009). In aqueous environments (lake, ocean), sonar-type methods can be used to detect bubble stream flow through water (e.g. Brewer et al. 2006). When high permeability zones are precisely located (well bores and faults), atmospheric monitoring chambers can be displayed at the surface to detect any abnormal $\mathrm{CO}_{2}$ flux originating from the soil (e.g. Edwards \& Riggs 2003; Madsen et al. 2009). Most monitoring strategies also include the use of geophysical methods to perform time-lapse monitoring of various physical properties of the shallow subsurface, in an indirect way. Among them, geoelectrical methods are of particular interest since they show a good sensitivity to the nature, composition and repartition of fluids in the subsurface and because they exhibit a much lower cost of implementation than seismic methods, which are often used by the oil and gas industry.

To date, many studies have highlighted the capacity of the electrical resistivity tomography (ERT) method to detect a $\mathrm{CO}_{2}$ transfer in the subsurface. Some of them consisted of laboratory scale investigations (e.g. Nakatsuka et al. 2010; Breen et al. 2012) and others of field-scale experiments (e.g. Xue et al. 2006; Kiessling et al. 2010; Auken et al. 2014). Most of these studies emphasize the fact that the ERT signals show a good sensitivity to liquid/gas saturation levels, thus allowing the detection of saturation variations induced by the presence of a gaseous plume in the subsurface, that is, a $\mathrm{CO}_{2}$ leakage path (e.g. Bergmann et al. 2012; Carrigan et al. 2013). Other shows that the ERT methods are also capable of detecting $\mathrm{CO}_{2}$ and/or mineral dissolution in the saturating water if these processes bring significant changes to the saturating fluid electrical conductivity (EC; e.g. Peter et al. 2012; Vialle et al. 2014).

The spectral induced polarization method (SIP) is another geoelectrical method that allows a broader geophysical characterization of geomaterials since it consists in the simultaneous measurement of the electrical resistivity of the ground and of a parameter generally 
called phase that can be used to describe the capacitive properties of the ground. The SIP method consists of studying the magnitude of these two parameters and their dependence on the frequency of the injected electrical signal. In many cases, the additional information provided by this method permits a more complete and precise interpretation of the structural and mineralogical composition of the subsurface compared to ERT surveys alone (e.g. Kemna et al. 2004; Schmutz et al. 2011), and a better understanding of the interactions existing between the solid matrix and the saturating fluid. SIP parameters have been proved to be sensitive to the saturation level of rocks (e.g. Ulrich \& Slater 2004; Cosenza et al. 2007; Breede \& Kemna 2012) and the chemical composition of the saturating fluid (e.g. Lesmes \& Frye 2001; Skold et al. 2011; Vaudelet et al. 2011; Weller et al. 2011). Other studies have also shown that SIP signals could be related to structural parameters of the rock such as the main grain diameter (e.g. Leroy et al. 2008), the mean pore throat size (e.g. Scott \& Barker 2003; Binley \& Kemna 2005) and the specific surface area (e.g. Weller et al. 2010).

This paper aims to assess whether the additional information provided by SIP measurements make this method relevant for the monitoring of $\mathrm{CO}_{2}$ transfers in the subsurface, and for the implementation of SIP measurements as part of a monitoring network. To the best of our knowledge, Dafflon et al. (2013) were the first to apply induced polarization measurements to monitor a $\mathrm{CO}_{2}$ intrusion in the shallow subsurface. However, they limited their study to the case of an intrusion of water saturated with dissolved $\mathrm{CO}_{2}$, neglecting the case of a gaseous $\mathrm{CO}_{2}$ transfer. Furthermore, the frequency dependence of SIP parameters was not studied.

In this paper, we present the results of a set of metric scale experiments, performed in controlled conditions, aiming at studying the impact of a gaseous $\mathrm{CO}_{2}$ injection on the SIP response of wellcharacterized unconsolidated granular media fully saturated with water. It is important to point out that our purpose is to study the capacity of the SIP method to detect a $\mathrm{CO}_{2}$ leakage in the shallow part of the subsurface, not its capacity to monitor the deep storage area itself. By working with a relatively simple medium, but still representative of the kind of material one can encounter in the subsurface (such as sandy aquifers), we aim to understand the first-order physicochemical phenomena induced by a $\mathrm{CO}_{2}$ leakage in the shallow subsurface and observe whether they may have a consequent impact on SIP parameter magnitude. In a companion paper (Kremer et al. 2016), we present an extended modeling work that brings valuable elements toward a better understanding of the experimental data presented here.

\section{MATERIALS AND METHODS}

\subsection{Spectral induced polarization}

In the subsurface, electrical current circulates thanks to the movement of ionic or electronic charges through conductive phases. Depending on the frequency of the electrical signal injected, current injection can lead to different polarization processes which basically result from the accumulation of these charges at the interface between the different components of the ground. In the low-frequency range $(f<100 \mathrm{~Hz})$, in the absence of metallic particles, the dominant mechanisms are the polarization of the electrical double layer that exists at the interface between the fluid and the mineral (e.g. Revil \& Florsch 2010), and the mechanism known as membrane polarization (polarization over multiple grain length; e.g. Marshall \& Madden 1959). For higher frequencies $\left(f>10^{3} \mathrm{~Hz}\right)$, the dominant polariza- tion mechanism becomes the Maxwell-Wagner polarization (e.g. Chen \& Or 2006a,b). It takes place at the interface between soil components that present different dielectric permittivity and EC values.

In the laboratory, SIP measurements can be performed using a four electrode setup, where an alternative electrical current of amplitude $I$ is injected through two electrodes and the potential difference $\Delta V$ is measured between the two other electrodes. If the studied medium polarizes, it results in a delay between the injected current and the measured voltage, which is generally expressed as an angle and therefore called the phase $\varphi$ (in radians). The complex conductivity $\sigma^{*}$ of the medium is then given by:

$\sigma^{*}=\left|\frac{I}{k \Delta V}\right| \exp (i \varphi)=|\sigma| \exp (i \varphi)$

where $k$ is a geometric factor linked to the position of the four electrodes and $i$ is the pure imaginary number.

The complex conductivity can be expressed as:

$\sigma^{*}=\sigma^{\prime}+i \sigma^{\prime \prime}$

where $\sigma^{\prime}$ is generally called the in-phase conductivity and $\sigma^{\prime \prime}$ is known as the quadrature conductivity. These two scalar quantities can be computed using the relations:

$|\sigma|=\left|\frac{I}{k \Delta V}\right|=\sqrt{\sigma^{\prime 2}+\sigma^{\prime \prime 2}}$

$\tan \varphi=\frac{\sigma^{\prime \prime}}{\sigma^{\prime}}$

In SIP studies, it is particularly interesting to work and reason with the in-phase conductivity $\sigma^{\prime}$ and the quadrature conductivity $\sigma^{\prime \prime}$ because these parameters reflect, respectively, the ohmic conduction properties of the sample and the capacitive properties and inductive response of the sample. In SIP studies, one important objective is to compute these two parameters and particularly to assess their magnitude and their dependence on the frequency of the injected signal.

At low frequencies $(f<100 \mathrm{~Hz})$, one of the main difficulties associated with the implementation of the SIP method is related to electrode stability over time. Indeed, the acquisition period being longer when the frequency is low, it is imperative to ensure that the SIP parameters measured on an unchanged system do not vary with time.

At intermediate frequencies $\left(10^{2}<f<10^{5} \mathrm{~Hz}\right)$, some parasitic effects can alter the measurements. They result from the electromagnetic (EM) inductive and capacitive coupling between the measuring and injecting cables and the studied sample (e.g. Hallof 1974; Zonge \& Wynn 1975; Pelton 1977). The magnitude of these effects depends mainly on the kind of cable used, their respective geometric display and the contact resistance of the electrodes (Zonge \& Hughes 1985). Note that we save the denomination 'high frequencies' for dielectric spectroscopy studies where the frequency of the injected signal is above $10^{5} \mathrm{~Hz}$.

Finally, another parasitic effect that can impact the signal measured over the whole frequency range results from polarization processes occurring at the interface between the electrode and the sample. If too great, these effects may overlap the response of the sample itself and complicate the interpretation of the measurements. Several experimental techniques can be applied to overcome them (e.g. Schwan 1968; Chelidze et al. 1999; Ishai et al. 2013), the main one being the use of non-polarizable electrodes instead of metal electrodes for potential measurements. 


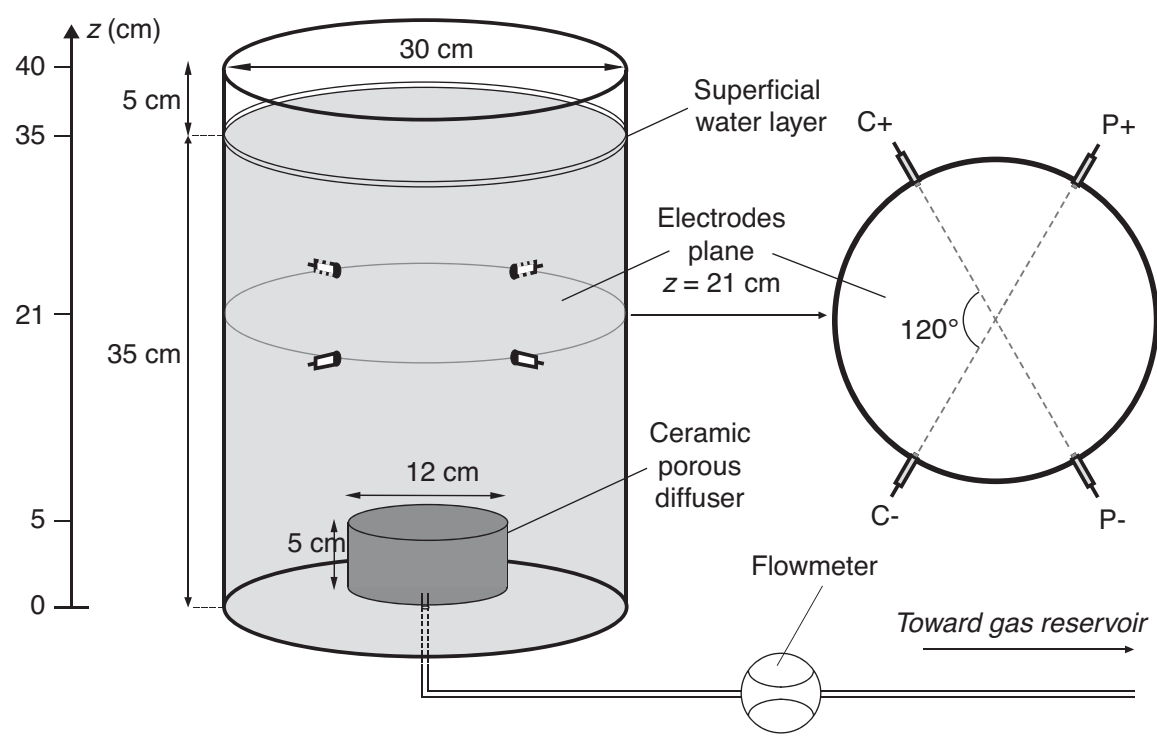

Figure 1. Sketch of the experimental setup. Gas injection is performed thanks to a porous diffuser located at the bottom of the cylindrical tank and is controlled by a flowmeter. Non-polarizable electrodes are located around the tank in threaded holes. C and P represent current and potential electrodes, respectively.

\subsection{Experimental setup}

All SIP measurements were performed using a SIP FUCHS III system (Radic Research). To conduct the experiments, we used a 40-cm-high, 30-cm-diameter cylindrical tank made of polyvinylchoride. Such dimensions allow investigation of a volume large enough with respect to the electrodes dimensions and spacing, and large enough to be representative of a field case, while keeping the tank filling and emptying process sufficiently short (about $1 \mathrm{hr}$ ) for daily experimentations. A hole is punched in the bottom of the tank to allow the connection between the gas inlet pipe and a 5-cm-high and 12-cm-diameter porous ceramic diffuser located at the bottom of the tank (Fig. 1). This diffuser enables a uniform and evenly spread distribution of the gas during the injection. Gas flow rate is controlled by a flowmeter with floating body located on the injection line.

The tank filling procedure was performed as follows for all the experiments. The container is first half filled with water. Next, the sand is deposited slowly using a sieve, whose mesh size is slightly larger than the largest grain size. When sand deposit reaches a 3$\mathrm{cm}$-thick layer, a manual packing procedure is applied, ensuring that the sand surface stays flat and that no air bubbles are trapped. Then another 3-cm-thick layer is added, packed with the same protocol, and so on, until reaching a sand height of $z=35 \mathrm{~cm}$. Throughout this procedure, sand is always underwater to ensure total saturation of the pore space. At the end of the sand packing procedure, the superficial water layer is removed, and only a thin free water layer of about $1 \mathrm{~mm}$ remains. We deliberately preserve this free water layer because monitoring its evolution helps us estimate the effective gas volume trapped in the medium during the experiment (gas invasion in the porous space triggers an increase of the superficial water layer thickness).

Non-polarizable electrodes are set up around the tank in threaded holes. For all the experiments, we used the same electrode configuration, as described in Fig. 1. Injection and measurement dipoles are located in the same plane at height $z=21 \mathrm{~cm}$. They are parallel, and each dipole forms a $120^{\circ}$ angle with the centre of the cylinder (Fig. 1). We chose this specific electrode setup after performing a numerical sensitivity study using the forward modeling software R3t (Binley 2015). Details of this study are given in Appendix A.1.
It allowed us to visualize the sensitivity distribution of different electrode configurations. In the end, we chose the ' $120^{\circ}$ configuration' because it is characterized by a particularly homogeneous sensitivity distribution. As we had no certainty about what would be the spatial repartition of the $\mathrm{CO}_{2}$ to be injected, we preferred a configuration showing a homogeneous sensitivity distribution.

To limit as much as possible parasitic effects resulting from the EM inductive coupling between the injecting and measuring cables, we used shielded coaxial cables for potential measurements. However, their extremity was not shielded and classical cables were used for current injection, which might still produce EM coupling effects. Several measurements were performed on silica sand fully saturated with water using a different cable display for each of them. All measurements produced the same results, indicating that the EM coupling effects are either negligible, or at least constant whatever the cable display.

\subsection{Non-polarizable electrodes}

As mentioned in Section 2.1, one of the sensitive issues of SIP measurements lies in the need to reduce as much as possible parasitic effects caused by polarization processes occurring at the electrodesample interface. For this purpose, we used custom-made $\mathrm{Cu} / \mathrm{CuSO}_{4}$ non-polarizable electrodes for both current injection and potential difference measurements (Fig. 2). A copper wire is immersed in a polyethylene tube filled with a saturated solution of $\mathrm{CuSO}_{4}$ solidified with gelatin. The electrode is closed at the outer end by a plug of cyanoacrylate glue, and is put in contact with the media through a porous filter (by Dionex) saturated with the electrolyte, that allows electrical current to flow through and preserves the integrity of the gelatinous mixture. We waited for at least $12 \mathrm{hr}$ before using the electrodes, to ensure that the gelatinous mixture was well solidified and that the electrode structure was therefore stable.

\subsubsection{Durability of the electrodes}

To estimate the durability of our electrodes, we monitored the contact resistance between two electrodes with an LCR-meter (ST2821A by Sourcetronic) everyday for a period of $6 \mathrm{~d}$. 


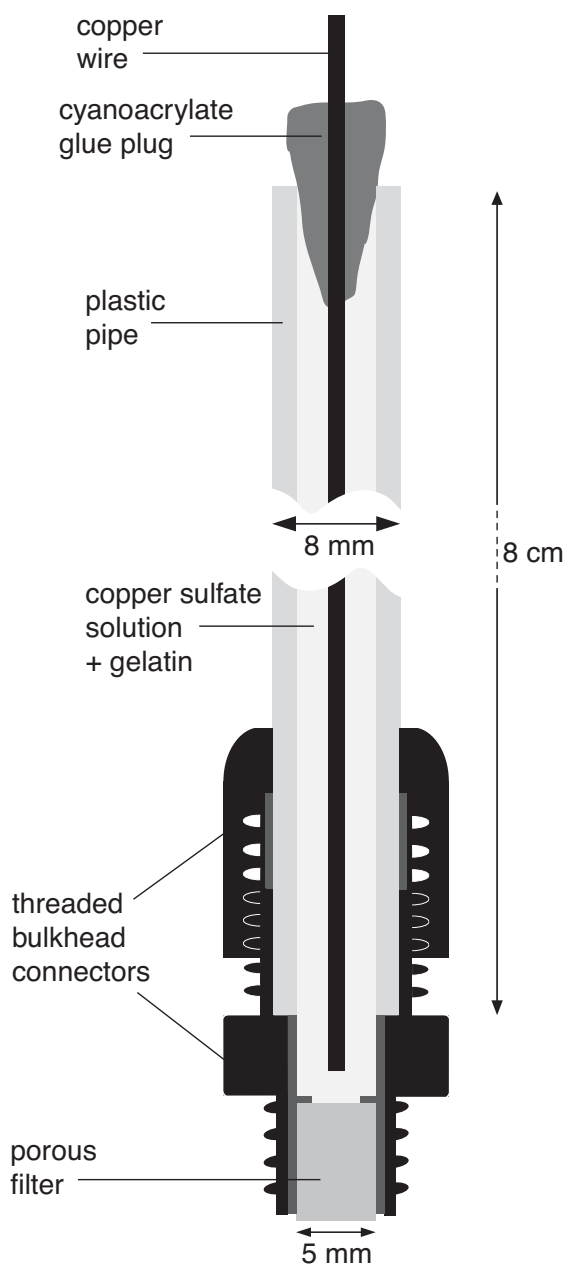

Figure 2. Sketch of the $\mathrm{Cu} / \mathrm{CuSO}_{4}$ non-polarizable electrodes.

Measured values remained in the range $0.9-1.3 \mathrm{k} \Omega$, which corresponds to the contact resistance of two lead-lead industrial chloridesodium/chloride Petiau electrodes (PMS9000 by SDEC) that are commonly used for self-potential and induced polarization field measurements. The physical integrity of our electrodes remained unchanged during this period: no air bubbles, leakage or oxidation of the copper wire was observed. Based on these observations, we chose to renew our set of electrodes every $5 \mathrm{~d}$ during the experimental campaign.

\subsubsection{Measurement on water}

The phase spectrum measured on water (Fig. 3), whose EC value is $273 \mathrm{mS} \mathrm{m}^{-1}$, exhibits values very close to 0 for frequencies below $100 \mathrm{~Hz}$, as expected from a non-polarizable material such as water. It means that the non-polarizing electrodes used are efficient and that no polarization phenomena occurred at the interface between the electrode and the electrolyte. However, non-zero phase values were measured at frequencies above $100 \mathrm{~Hz}$. This phenomenon is likely due to the strong impedance of the non-polarizing electrodes (Tirado et al. 2000; Abdulsamad et al. 2014; Huisman et al. 2014) and/or to EM inductive and capacitive coupling effects (Zonge \& Wynn 1975; Pelton 1977).

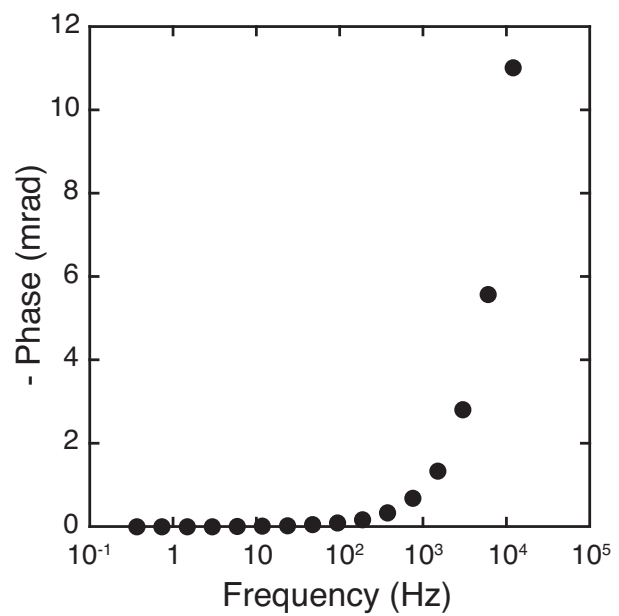

Figure 3. Phase spectra measured on cylindrical tank filled with only water with an electrical conductivity value of $273 \mathrm{mS} \mathrm{m}^{-1}(\mathrm{NaCl})$. Phase values are very close to zero for frequencies below $100 \mathrm{~Hz}$ and increase progressively for frequencies above $100 \mathrm{~Hz}$.

\subsubsection{Geometrical factor stability test}

To assess the ability of our electrodes to measure electrical potentials and inject electrical current, we performed resistivity measurements using the same cylindrical tank used during the experiment, filled with water of known EC. Various water EC values were used, and measurements were performed using non-polarizable electrodes and stainless steel electrodes. For each acquisition the geometric factor associated with the $120^{\circ}$ electrode configuration was calculated. Its value was also estimated through a modeling work, using the inverse and forward modeling code R3t (Binley \& Kemna 2005). All the measured and simulated geometric factors show similar values, with a mean measurement uncertainty of 1.8 per cent. We therefore estimate that our non-polarizable electrodes have a good capability for injecting electrical current and measuring potential differences.

\subsubsection{Measurement stability over time}

We evaluated the stability of our electrodes through time by performing several successive SIP measurements on a media similar to those studied during the experimental campaign. The cylindrical tank was filled with pure silica sand fully saturated with water whose EC value was calibrated to $40 \mathrm{mS} \mathrm{m}^{-1}$ by dissolving $\mathrm{NaCl}$ into deionized water. This value corresponds approximately to the EC of drinking water. We waited $24 \mathrm{hr}$ to allow the medium to reach chemical equilibrium, and performed successive SIP measurements during a period of $5 \mathrm{hr}$. All values of the measured phase and bulk conductivity $\left(\sigma_{\text {bulk }}=|\sigma|\right)$ were stable over time for the whole frequency range (Fig. 4). Bulk conductivity values were all between 12.4 and $12.5 \mathrm{mS} \mathrm{m}^{-1}$ (which is equivalent to 80.6 and $80 \Omega \mathrm{m}$ in terms of resistivity), and phase value variations were always lower than $0.2 \mathrm{mrad}$, whatever the frequency of the injected signal, indicating a good measurement repeatability (Fig. 4b).

\subsection{Materials}

Experiments were performed with two types of sand. The first one was a natural siliceous sand composed of 98 per cent mass of silica and 2 per cent mass of feldspars and micas. Through sieving 
(a)

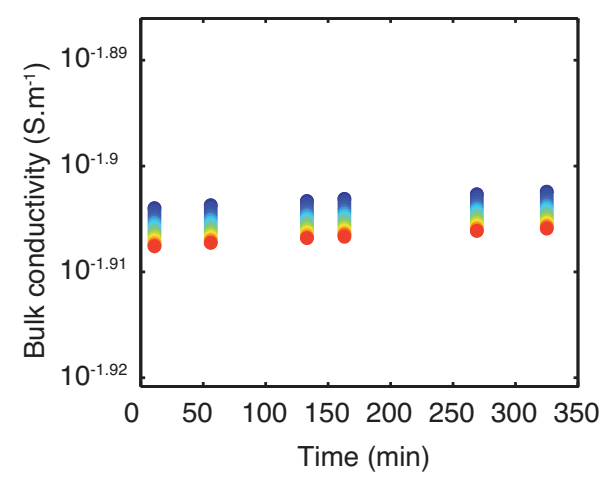

(b)

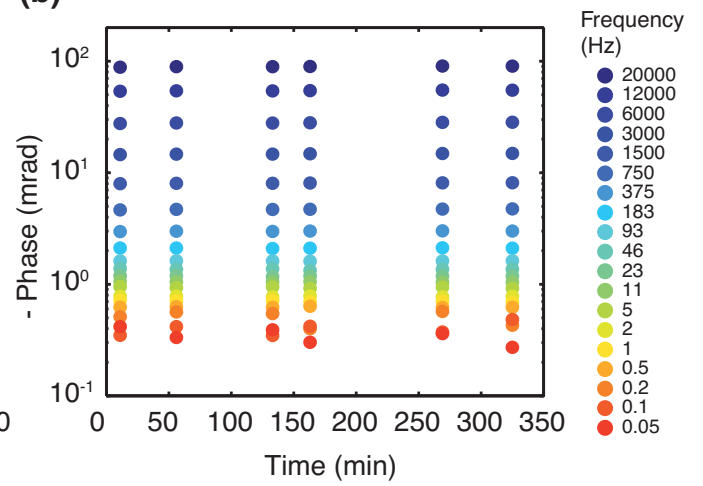

Figure 4. Stability along time of measurements on silica sand fully saturated with water whose electrical conductivity is calibrated to $40 \mathrm{mS} \mathrm{m}^{-1}$. Each colour represents a given frequency in the range $0.05 \mathrm{~Hz}-20 \mathrm{kHz}$. (a) Bulk conductivity values. (b) Phase values.

Table 1. Summary of the experimental parameters relative to each of the 12 experiments performed.

\begin{tabular}{lccc}
\hline Experiment number & Sand type & Gas type & Water $\mathrm{EC} \sigma_{\mathrm{w}}\left(\mathrm{mS} \mathrm{m}^{-1}\right)$ \\
\hline E1 & Silica & $\mathrm{N}_{2}$ & 15 \\
E2 & Silica & $\mathrm{N}_{2}$ & 40 \\
E3 & Silica & $\mathrm{N}_{2}$ & 100 \\
E4 & Silica & $\mathrm{CO}_{2}$ & 15 \\
E5 & Silica & $\mathrm{CO}_{2}$ & 40 \\
E6 & Silica & $\mathrm{CO}_{2}$ & 100 \\
E7 & Carbonate & $\mathrm{N}_{2}$ & 15 \\
E8 & Carbonate & $\mathrm{N}_{2}$ & 40 \\
E9 & Carbonate & $\mathrm{N}_{2}$ & 100 \\
E10 & Carbonate & $\mathrm{CO}_{2}$ & 15 \\
E11 & Carbonate & $\mathrm{CO}_{2}$ & 40 \\
E12 & Carbonate & $\mathrm{CO}_{2}$ & 100 \\
\hline
\end{tabular}

operations, we restricted the grain size distribution to the range $100-250 \mu \mathrm{m}$. Porosity and permeability (to water) were estimated using a macro-permeameter device whose functioning is described in Clavaud (2001). Measured porosity value was 0.42 and the mean permeability to water obtained with a set of three measurements was $12.1 \pm 0.3 \mathrm{D}$. The second sand is an artificial carbonate sand whose molal composition is 98 per cent calcium carbonate $\left(\mathrm{CaCO}_{3}\right)$ and 2 per cent magnesium carbonate $\left(\mathrm{MgCO}_{3}\right)$. Grain size ranged between 50 and $400 \mu \mathrm{m}$, measured porosity was 0.32 and permeability to water was estimated to be $4 \pm 0.33 \mathrm{D}$.

The initial EC of the saturating water was controlled by dissolving $\mathrm{NaCl}$ into deionized water. Three values were used during the experiments: 15,40 and $100 \mathrm{mS} \mathrm{m}^{-1}$ corresponding, respectively, to the $\mathrm{EC}$ of fresh water, drinking water and low concentration brine. Two types of gas were used during the experiments. Nitrogen $\left(\mathrm{N}_{2}\right)$, which presents a very low solubility in water $(0.016$ volume per volume at a temperature of $\left.0{ }^{\circ} \mathrm{C}\right)$, and does not react with the solid phase; and carbon dioxide $\left(\mathrm{CO}_{2}\right)$, whose solubility in water at $0{ }^{\circ} \mathrm{C}$ is 0.88 and which can react with the solid matrix. Our experimental campaign consists of combining all the parameters described previously, which resulted in the realization of 12 different experiments, denoted from E1 to E12, whose experimental parameters are summarized in Table 1.

These different experiments are designed to study in the laboratory several cases that could be encountered in the field. Experiments with carbonate and silica sand can respectively bring insights about the electrical response of $\mathrm{CO}_{2}$ leakage into a calcareous or a sandy aquifer. Using three values for the saturating water, EC allows consideration of three types of water that could be encountered in the ground (fresh, drinking and low concentration brine). Finally, gaseous $\mathrm{CO}_{2}$ flowing through earth material can either dissolve into the saturating water (e.g. Carrigan et al. 2013) or invade the porous space in gaseous form and locally decrease the water saturation level (e.g. Auken et al. 2014). Thus, experiments involving gaseous $\mathrm{N}_{2}$, which cannot dissolve in water, serve as a reference experiment to study the effect of water saturation variations alone, without considering dissolution processes.

\subsection{Experimental protocol}

Every experiment was carried out as follows. The tank filling procedure was performed $12 \mathrm{hr}$ before the beginning of the experiment in order to allow the studied medium to get as close as possible to a chemical equilibrium state. A reference acquisition was performed before the beginning of gas injection, to obtain the initial SIP parameter values. Then, gas was injected first at a flow rate $Q_{1}=3 \mathrm{~L} \mathrm{~h}^{-1}$ during a period of between 2 and $6 \mathrm{hr}$, depending on the experiment, while SIP acquisitions were performed regularly (with a time interval ranging between $5 \mathrm{~min}$ and $1 \mathrm{hr}$ depending on the experiment). The gas injection rate was then increased to $Q_{2}=8 \mathrm{~L} \mathrm{~h}^{-1}$ for a period of $1-2 \mathrm{hr}$, and acquisitions were performed regularly until the stopping of gas injection, that is, the end of the experiment.

During all experiments, the effective gas saturation level in the media was estimated by monitoring the increase of the superficial water layer thickness (see Fig. 1). The chemical composition of the saturating water was also monitored. Water samples were collected from the medium three times during the experiment: before the beginning of gas injection, before the increase of the injection rate, and at the end of the experiment. The samples were extracted from the centre of the media using a thin graduated pipette plunged into the tank, and were submitted to chromatographic analysis, and $\mathrm{pH}$ and EC measurements (Table 2). Additional measurements were performed to ensure that water-sampling processes (plunging and removing the pipette, and water extraction) did not change the measured phase and resistivity values.

Note that these experiments were conducted on an open system, which means that when the gas reached the surface of the sand cylinder, it escaped directly into the atmosphere and did not accumulate in the medium. Given the relatively high permeability of the sands used, gas migration up to the surface occurred quite quickly. In about $30 \mathrm{~s}$ to $1 \mathrm{~min}$, the gas migration pathways were set and 
Table 2. Water EC, $\mathrm{pH}$ and ionic concentrations obtained from the analysis of water samples taken during experiments E10, E11 and E12. The value of $\left[\mathrm{HCO}_{3}{ }^{-}\right]$corresponds to the total dissolved carbonate species concentrations.

\begin{tabular}{lccccc}
\hline Experiment & Time (h) & $\begin{array}{c}\sigma_{\mathrm{f}} \\
\left(\mathrm{mS} \mathrm{m}^{-1}\right)\end{array}$ & $\mathrm{pH}$ & $\begin{array}{c}{\left[\mathrm{HCO}_{3}{ }^{-}\right]} \\
\left(\mathrm{mmol} \mathrm{L}^{-1}\right)\end{array}$ & $\begin{array}{c}{\left[\mathrm{Ca}^{2+}\right]} \\
\left(\mathrm{mmol} \mathrm{L}^{-1}\right)\end{array}$ \\
\hline \multirow{3}{*}{ E10 } & 0 & 27.0 & 8.70 & 0.49 & 0.45 \\
& 4.5 & 40.2 & 7.21 & 1.23 & 0.68 \\
& 7 & 65.0 & 6.21 & 13.0 & 5.75 \\
E11 & 0 & 51.1 & 8.80 & 0.22 & 0.31 \\
& 7 & 105 & 6.07 & 7.37 & 8.04 \\
& 9 & 110 & 6.02 & 7.94 & 7.66 \\
E12 & 0 & 125 & 8.55 & 0.36 & 0.40 \\
& 7 & 170 & 6.19 & 16.4 & 8.18 \\
& 9.5 & 190 & 6.17 & 22.4 & 6.98 \\
\hline
\end{tabular}

relatively stable. From this moment on, the effective gas saturation level in the media did not change until the injection rate was increased.

\section{RESULTS}

\subsection{Reference acquisition}

The SIP parameters spectra measured at the initial state on two media composed of carbonate sand or silica sand (Fig. 5), both fully saturated with water of identical EC value $\left(\sigma_{\mathrm{w}}=40 \mathrm{mS} \mathrm{m}^{-1}\right)$, are of good quality. Several measurements at the initial state were performed to study the stability of the acquisition. They are not shown here, but demonstrated an excellent repeatability, comparable to the data presented in Fig. 4. Even at low fre- quency $(f<1 \mathrm{~Hz})$, the repeatability is very good despite what the high error bars calculated by the acquisition device suggest. Note that Weller et al. (2011) showed that the error bars produced by the SIP FUCHS III system (Radic Research) are overestimated.

The bulk conductivity values measured are similar whatever the nature of the sand (between 12.3 and $12.6 \mathrm{mS} \mathrm{m}^{-1}$, which correspond to resistivity values of respectively 81.3 and $79.4 \Omega \cdot \mathrm{m}$, Fig. 5a). A slightly stronger dependence on frequency is observed for the silica sand, for which the percentage frequency effect (see definition in Lesmes \& Frye 2001) is 0.88 per cent versus 0.15 per cent for the carbonate sand. The shape of the phase spectra is similar for both sands (Fig. 5b), but a clear shift is observed between the two spectra for the low-frequency part $(f<100 \mathrm{~Hz})$. In this frequency domain, phase values are around $0.1 \mathrm{mrad}$ for the carbonate sand and around $1 \mathrm{mrad}$ for the silica sand. In both cases, these values are lower than or equal to the limit resolution of the SIP FUCHS III measuring device, which is around $1 \mathrm{mrad}$ according to Radic (2004). These low values indicate that little information can be retrieved from the low-frequency part of the phase spectra, except that polarization processes have a weak magnitude in this frequency range. For higher frequencies $(f>100 \mathrm{~Hz})$, a better confidence in the data is obtained since measured values are always higher than $2 \mathrm{mrad}$.

From the measured conductivity and phase values, we derived the in-phase conductivity $\sigma^{\prime}$ and the quadrature conductivity $\sigma^{\prime \prime}$ spectra (Figs 5c and d). The in-phase conductivity spectra and the quadrature conductivity spectra have a very similar shape to the bulk conductivity spectra and the phase spectra respectively. These similarities indicate that the measured phase values are mainly representative of the capacitive properties and inductive response of the medium and that the bulk conductivity values are mainly related to ohmic conduction.

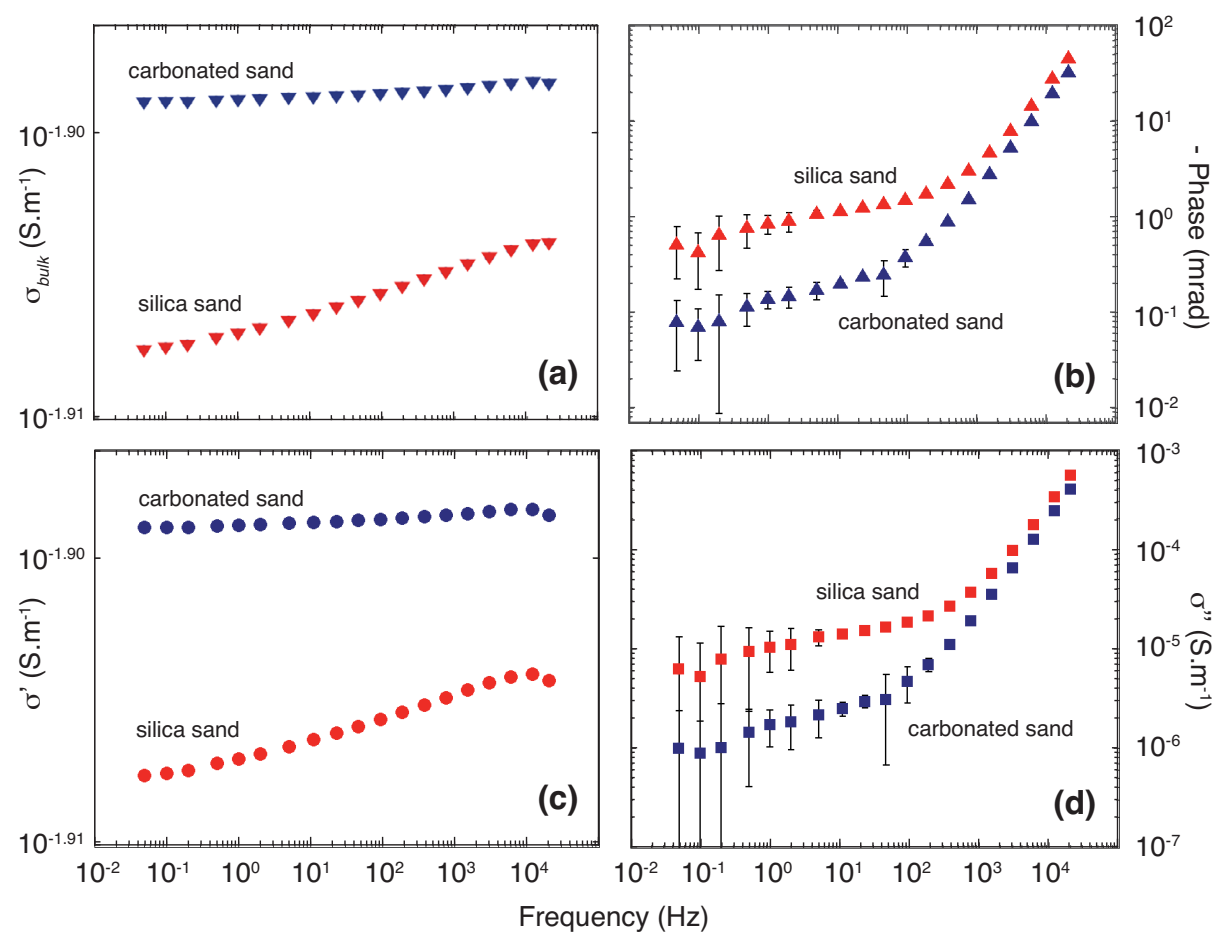

Figure 5. Measured and calculated SIP parameters obtained from acquisitions on silica sand and carbonate sand, both fully saturated with water whose electrical conductivity is $40 \mathrm{mS} \mathrm{m}^{-1}$. (a) Measured bulk conductivity spectra. (b) Measured phase spectra. (c) Calculated in-phase conductivity spectra. (d) Calculated quadrature conductivity spectra. 


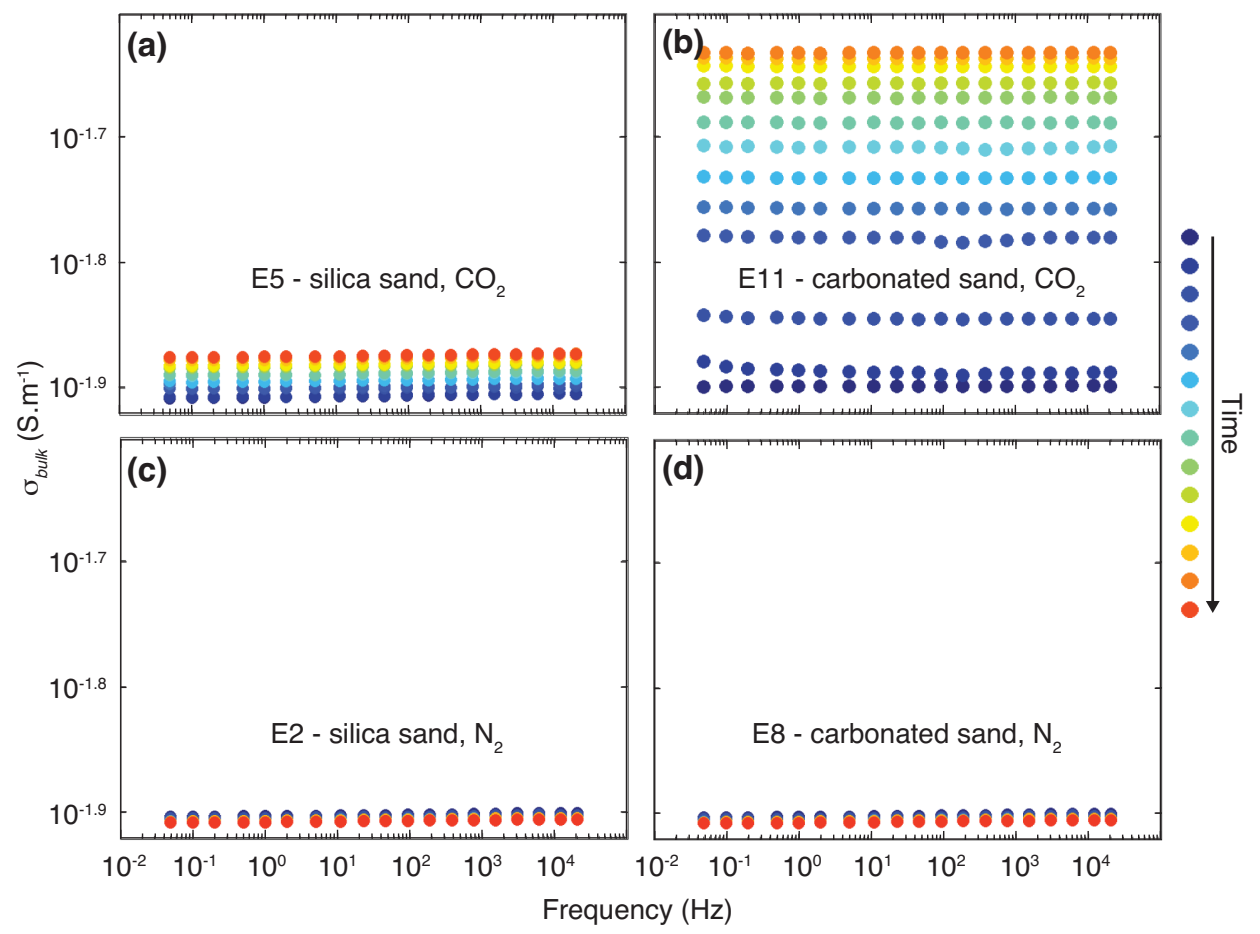

Figure 6. (a)-(d) Evolution of bulk conductivity spectra during experiments E5, E11, E2 and E8. Cold colours correspond to the spectra measured at the beginning of the experiment. Colours evolve from blue to red as the experiment advances in time.

\subsection{Signal evolution during gas injection}

\subsubsection{Bulk conductivity}

All the bulk conductivity spectra measured during experiments E2, E5, E8 and E11 (Fig. 6) are flat, showing that $\sigma_{\text {bulk }}=|\sigma|$ is nearly independent of frequency. The magnitude of $\sigma_{\text {bulk }}$ decreases very slightly during experiments E2 and E8 (Figs $6 \mathrm{c}$ and d), for which the injected gas is $\mathrm{N}_{2}$. During experiments $\mathrm{E} 5$ and $\mathrm{E} 11\left(\mathrm{CO}_{2}\right.$ injection), $\sigma_{\text {bulk }}$ follows an increasing trend with time, which is stronger when the studied material is carbonate sand (Fig. 6d).

\subsubsection{Phase evolution during experiments}

As shown previously (Fig. 5b), the phase response of the studied medium depends on the frequency of the injected signal. Figs 7 and 8 show the evolution of the phase spectra during gas injection for the experiments E2 and E11, respectively. We chose these two experiments as examples because they represent the two 'extreme' cases in terms of gas-sand system reactivity. E2 involves a nearly totally non-reactive system whereas E11 is the most potentially reactive system. The considerations that follow are however observable on any of the 12 experiments. We see from these figures that the phase magnitude evolution is also dependent on the frequency of the injected signal. Three frequency domains showing three different behaviours can be distinguished.

At very low frequencies $(f<1 \mathrm{~Hz})$, no clear trend can be observed for either of the experiments, the phase values measured during the experiments are dispersed randomly.

For what we call the low frequencies $(1<f<100 \mathrm{~Hz})$, the observed variations are weak (e.g. Fig. 7) and seem also partly random. The variation pattern between two neighbouring frequencies is not always the same (e.g. Figs 7 and 8, left subplots). In this frequency range, we are again confronted with 'erratic behaviour'.
We call the third frequency domain 'intermediate' $\left(10^{2}<f<2.10^{4} \mathrm{~Hz}\right)$, saving the denomination 'high frequency' for dielectric spectroscopy studies where the frequency of the injected signal is above $10^{5} \mathrm{~Hz}$. In the intermediate-frequency range, the phase values always follow a systematic variation pattern (Figs 7 and 8, right subplots). Measurement uncertainty is very weak in this frequency range. We can therefore consider that the observed trends result from a physical modification of the studied media triggered by the gas injection.

\subsubsection{Evolution of $\sigma^{\prime}$ and $\sigma^{\prime \prime}$ at intermediate frequencies}

In this section, we show the evolution over time of the medium in-phase conductivity $\sigma^{\prime}$ and of the quadrature conductivity $\sigma^{\prime \prime}$ calculated for intermediate frequencies. Parameter $\sigma^{\prime \prime}$ is mainly representative of the capacitive properties and the inductive response of the medium, and therefore allows close monitoring of the evolution of the polarization processes magnitude.

The evolution in time of $\sigma^{\prime}$ and $\sigma^{\prime \prime}$ values measured at the frequency $f=12 \mathrm{kHz}$, for all the experiments, is shown in Fig. 9. In each graph, the first orange vertical line on the left indicates the beginning of gas injection (at a rate of $3 \mathrm{~L} \mathrm{~h}^{-1}$ ), and the second one indicates the increase of the gas injection rate from 3 to $8 \mathrm{~L} \mathrm{~h}^{-1}$. We note that $\sigma^{\prime}$ and $\sigma^{\prime \prime}$ globally evolve in the same way, either decreasing or increasing together. A diminution of the conductive properties of the media is systematically accompanied by a diminution of the capacitive properties, and vice versa if the parameters increase. Three different behaviours can be observed.

When the injected gas is $\mathrm{N}_{2}$ (Figs 9a, b, c, g, h and i), an inert gas, $\sigma^{\prime}$ and $\sigma^{\prime \prime}$ decrease quickly after the gas injection starts, and keep decreasing during the experiment, sometimes with a stronger fall when the gas injection rate is increased. In most cases, $\sigma^{\prime}$ and $\sigma^{\prime \prime}$ values tend to stabilize when approaching the end of the experiment. 


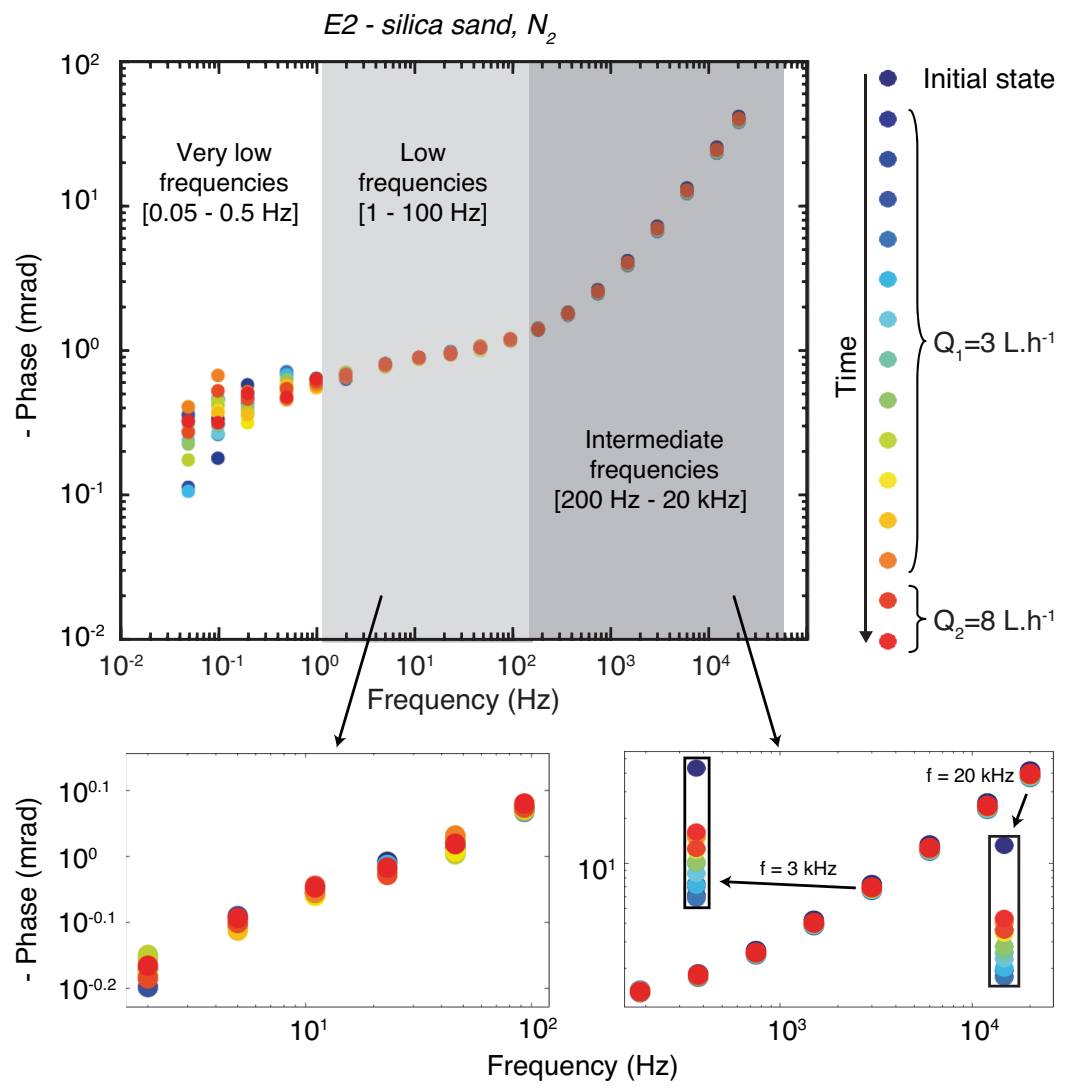

Figure 7. Phase spectra evolution during experiment E2. Spectra colours evolve from blue to red as the experiment advances in time. Subplots are, respectively, zooms on the low and intermediate-frequency ranges. The two black boxes in are zooms showing the phase evolution pattern for frequencies 3 and $20 \mathrm{kHz}$.

When the injected gas is $\mathrm{CO}_{2}$ and the sand studied is composed of carbonates (Figs $9 \mathrm{j}-1$ ), $\sigma^{\prime}$ and $\sigma^{\prime \prime}$ increase continuously during the experiment.

Finally, when the injected gas is $\mathrm{CO}_{2}$, and the sand is composed of silica (Figs 9d-f), a hybrid behaviour is observed. Parameters $\sigma^{\prime}$ and $\sigma^{\prime \prime}$ drop quickly after the beginning of $\mathrm{CO}_{2}$ injection, after which they both follow an increasing trend until the end of the experiment.

\section{DISCUSSION}

\subsection{Results interpretation}

The stability of the measurements with time has been demonstrated in Section 2.3 on an unchanging media (Fig. 4). Considering that the instrumental setup remains the same during the experiment, it follows that all the signal variations observed are necessarily related to physicochemical variations occurring inside the studied media.

During experiments involving the injection of $\mathrm{N}_{2}$, which is a gas nearly non-soluble in water, the main trend observed is a decrease of the measured in-phase conductivity and of the quadrature conductivity. It is then assumed that this evolution should be attributed to the diminution of the water saturation level caused by gaseous $\mathrm{N}_{2}$ invasion in the pore space (invasion of a resistive phase). Such behaviour has been reported in many published studies (e.g. Ulrich \& Slater 2004; Binley et al. 2005; Cosenza et al. 2007; Jougnot et al. 2010; Revil 2013).

For experiments E7, E8 and E9 $\left(\mathrm{N}_{2}\right.$ into carbonate sand), we monitored the $\mathrm{EC}$ value of the water to assess whether carbonate dissolution was occurring during the experiment. Variations are always lower than 3 per cent, indicating that dissolution processes are very weak, hence supporting the hypothesis that in the case of an $\mathrm{N}_{2}$ injection, it is the water saturation level variations that control the diminution of $\sigma^{\prime}$ and $\sigma^{\prime \prime}$. This assumption is supported by visual observation. In each experiment, a slight increase of the superficial water layer thickness can be observed quickly after the beginning of gas injection, and also sometimes when the gas injection rate is increased. This elevation implies that part of the porous space has been invaded by gas, reducing the effective water saturation level $S_{\text {eff }}$. According to our observations, $S_{\text {eff }}$ does not evolve much during the experiments. When the main gas pathways are set up and have reached the surface, $S_{\text {eff }}$ stabilizes. We estimate, for every experiment, the mean effective saturation level reached when the medium is stabilized to be 98 per cent, which is very low.

For experiments E10, E11 and E12 (injection of $\mathrm{CO}_{2}$ ) into carbonate sand, the main behaviour is a continuous increase of the conductivities $\sigma^{\prime}$ and $\sigma^{\prime \prime}$. Chemical analysis performed on water samples extracted from the studied media at regular intervals showed that strong dissolution processes are occurring (see Table 2). The injected $\mathrm{CO}_{2}$ is progressively dissolving in the saturating water, leading to its acidification, which itself enhances the dissolution of carbonates, as shown by the increasing concentration of $\mathrm{Ca}^{2+}$ ions. As increasing ionic concentrations necessarily leads to increasing in-phase conductivity, we can safely attribute the observed increasing trend followed by $\sigma^{\prime}$ and $\sigma^{\prime \prime}$ to the dissolution processes that occur. These aspects are consistent with many published studies reporting an increase of the quadrature conductivity $\sigma^{\prime \prime}$ with increasing water EC (e.g. Garrouch \& Sharma 1994; Lesmes \& Frye 2001; Revil \& Skold 2011; Weller et al. 2011). 
E11 - carbonated sand, $\mathrm{CO}_{2}$

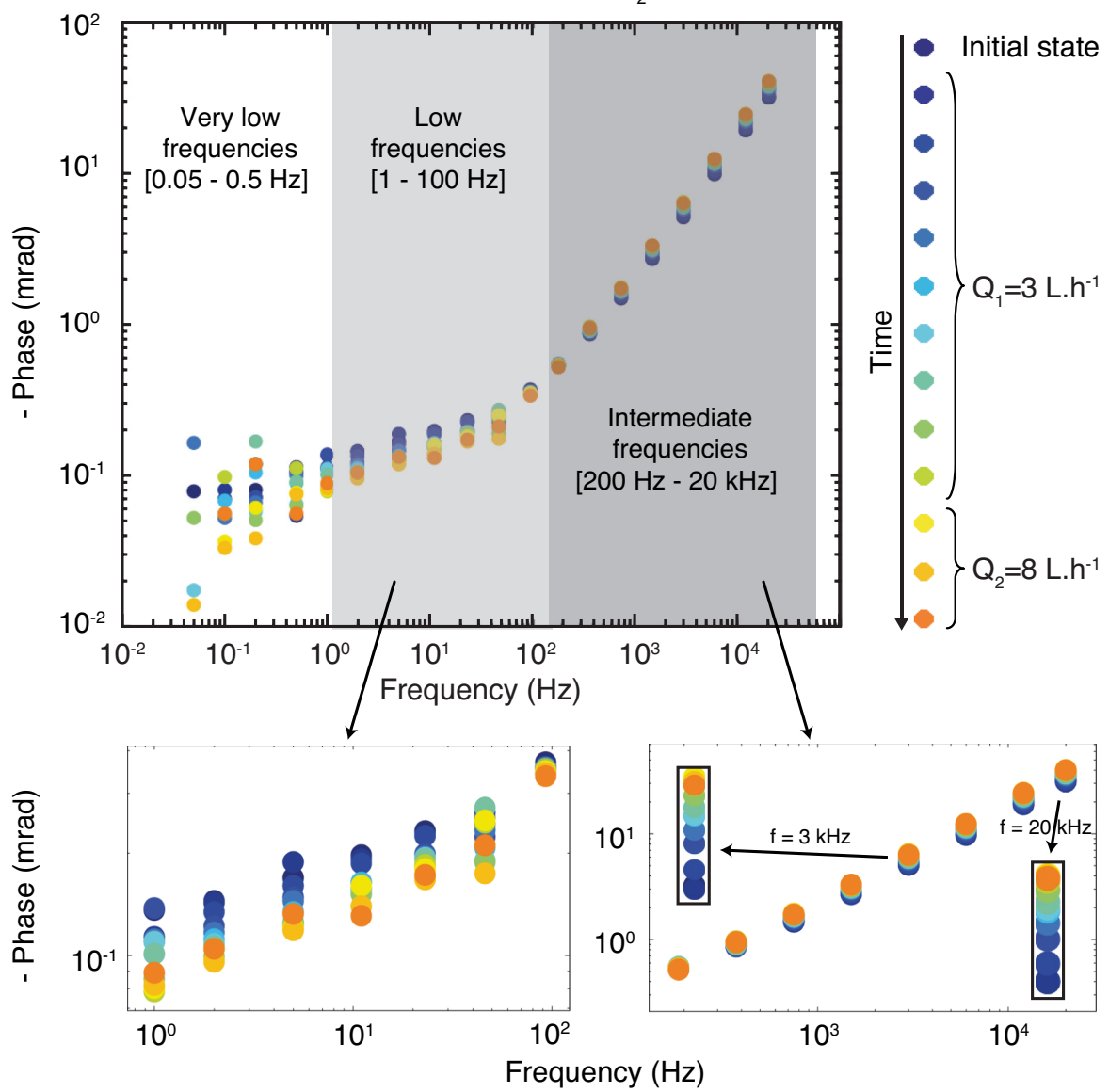

Figure 8. Phase spectra evolution during experiment E11. Spectra colours evolve from blue to red as the experiment advances in time. Subplots are, respectively, zooms on the low and intermediate-frequency range. The two black boxes in are zooms showing the phase evolution pattern for frequencies 3 and $20 \mathrm{kHz}$.

These considerations also explain the hybrid behaviour observed for experiments $\mathrm{E} 4, \mathrm{E} 5$ and $\mathrm{E} 6\left(\mathrm{CO}_{2}\right.$ injection into saturated silica sands). A drop of $\sigma^{\prime}$ and $\sigma^{\prime \prime}$ values is observed quickly after the beginning of the experiment. Then, as the $\mathrm{CO}_{2}$ progressively dissolves into water, $\sigma^{\prime}$ and $\sigma^{\prime \prime}$ follow an increasing trend. Silica being nearly non-soluble in water for this $\mathrm{pH}$ range, a lower amount of ionic species is brought to solution than with the carbonate sand, which explains why the magnitude of the increase is lower with silica sands than with carbonate sand.

\subsection{Magnitude of variations}

As shown previously, the reduction of the water saturation level in the media due to gas injection results in a decrease of the in-phase and quadrature conductivity values. When significant dissolution processes occur, for instance due to the dissolution of $\mathrm{CO}_{2}$ and/or carbonate sand, it results in an increase of both $\sigma^{\prime}$ and $\sigma^{\prime \prime}$ values. These two behaviours occur with different magnitudes. Fig. 10 shows the evolution of $\sigma^{\prime}$ and $\sigma^{\prime \prime}$ values measured at the frequency $f=12 \mathrm{kHz}$ during experiments $\mathrm{E} 2, \mathrm{E} 5, \mathrm{E} 8$ and E11, that is, for each of the four sand/gas systems studied. These figures highlight the fact that variations are much stronger during experiments involving carbonate sand and $\mathrm{CO}_{2}$, that is, when important dissolution processes are involved. Changes caused by desaturation processes are much lower and are hardly visible. This last point could likely be linked to the fact that desaturation processes are not very significant. Ac- cording to our calculations, the minimal bulk water saturation level reached during these experiments is never lower than 0.98 (maximum reduction of 2 per cent).

In any case, it is important to note that despite their very different magnitudes, the variations of $\sigma^{\prime \prime}$ due to dissolution or desaturation processes all correspond to phase variations at least equal and superior to $2 \mathrm{mrad}$, which is twice the resolution limit of our measuring device. This indicates that the capacitive properties of the medium are indeed modified due to physical processes (desaturation and dissolution), and are not the result of random instrumental effects.

\subsection{Additional information provided by SIP measurements}

As expected, the bulk conductivity $\sigma_{\text {bulk }}$ classically measured by resistivity surveys is also affected by the physical modifications of the medium caused by gas injection. However, another important observation can be made when comparing the magnitude of the relative changes sustained by $\sigma_{\text {bulk }}$ and $\sigma^{\prime \prime}$ parameters. Even though they systematically evolve in the same direction, it can be noted that the quadrature conductivity $\sigma^{\prime \prime}$ (measured at the frequency $f=12 \mathrm{kHz}$ ) is always more affected by the physicochemical phenomenon occurring in the medium than is the bulk conductivity. Fig. 11 highlights this point for experiments where the main physical process occurring is desaturation (Fig. 11a) as well as for experiments where the main processes are dissolution processes (Fig. 11b). 

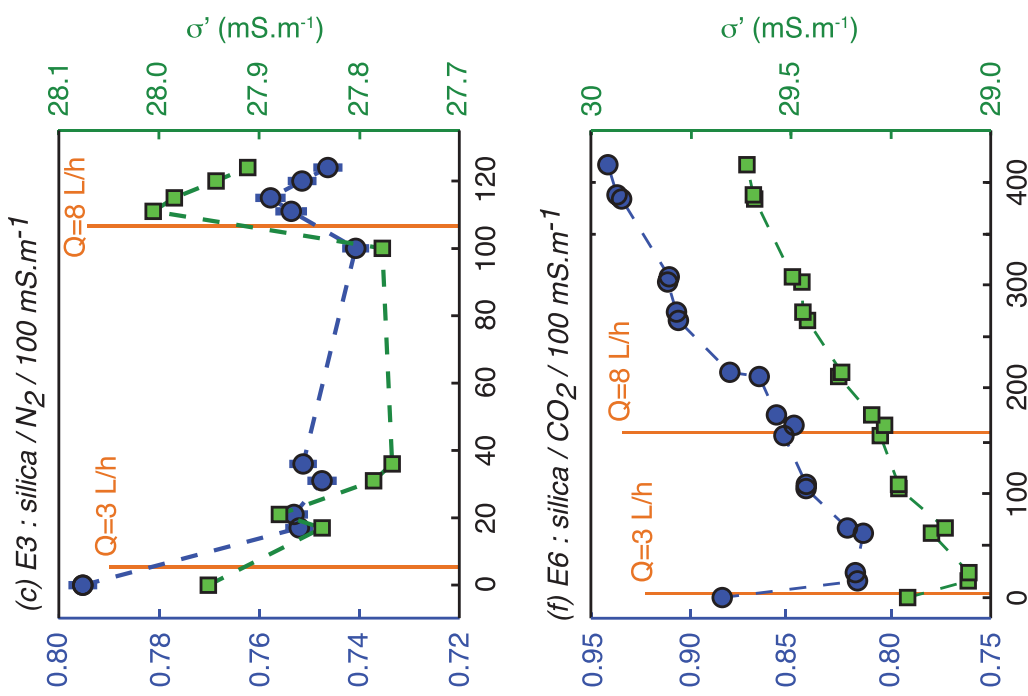

กั่
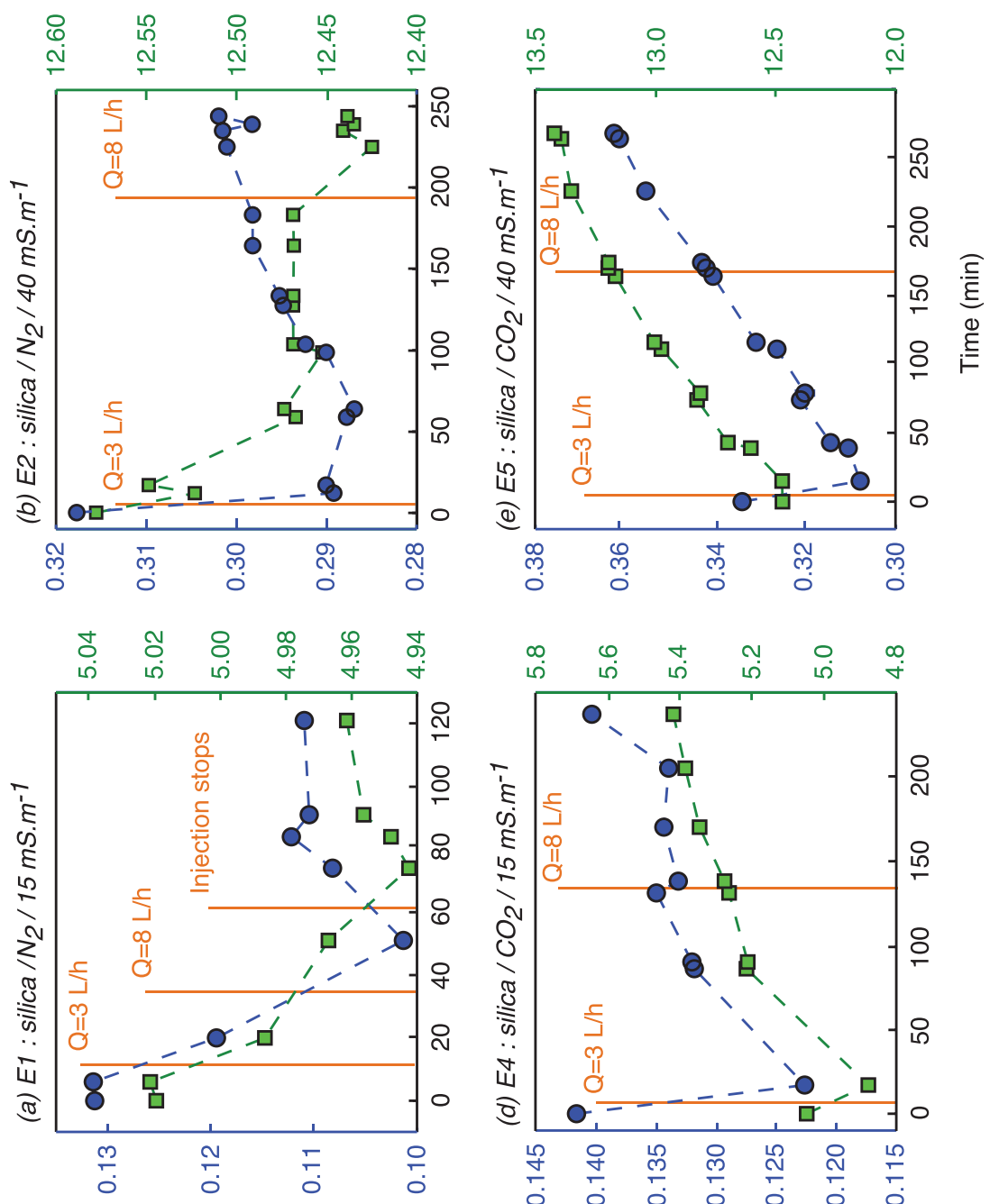

(1-m.sm) ،

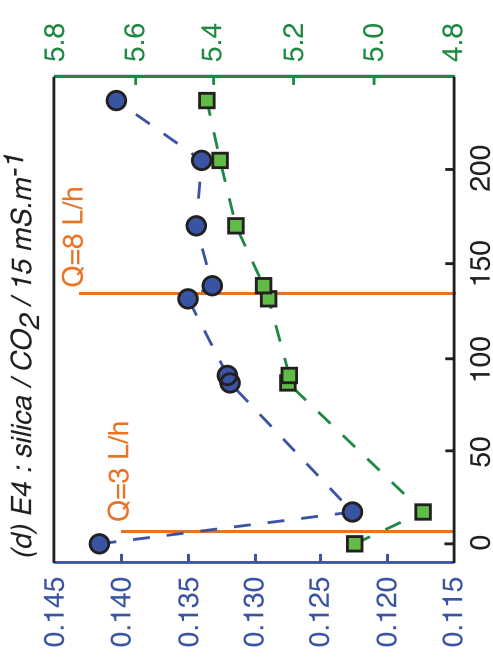

(1.m? 

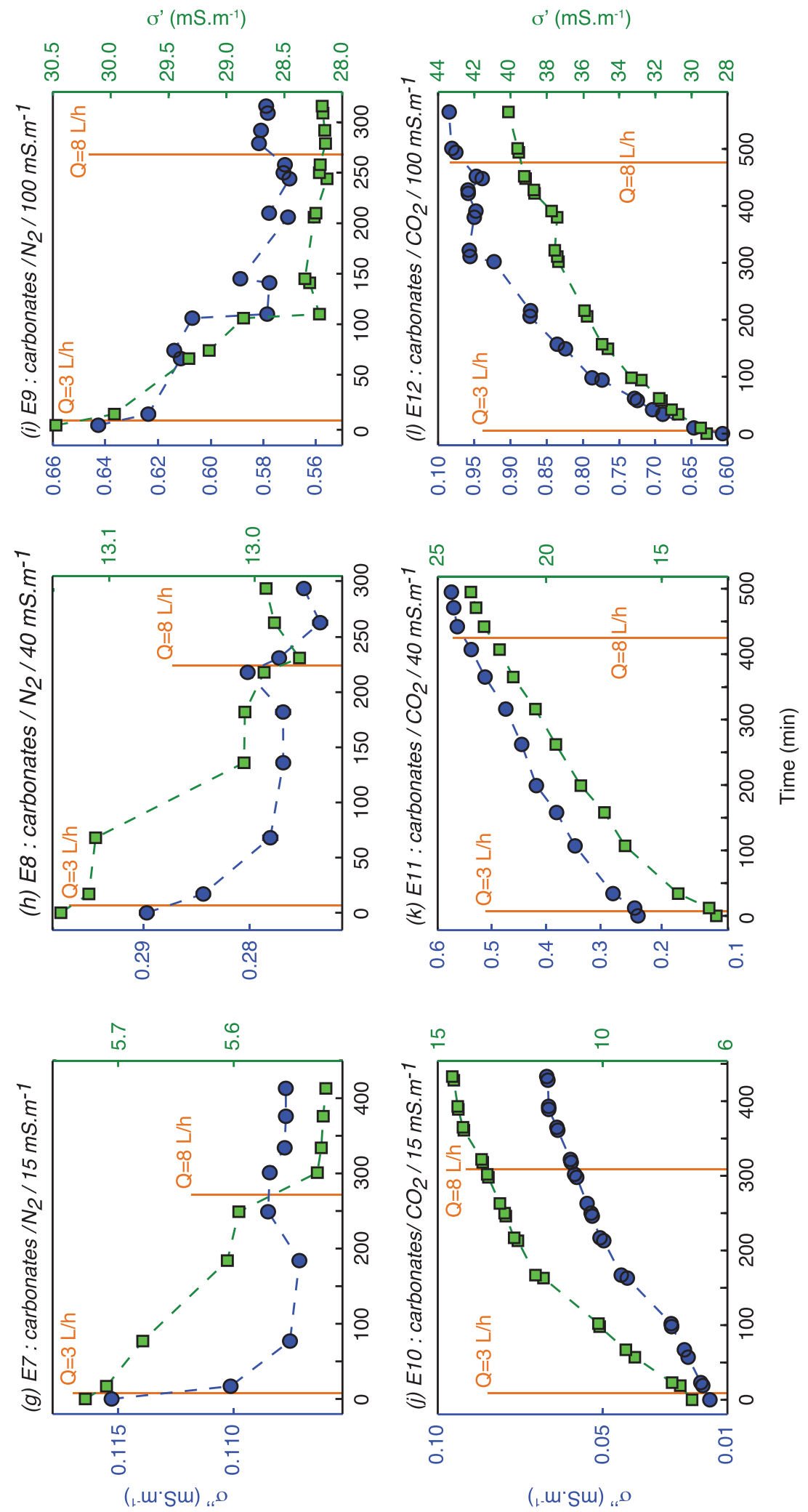


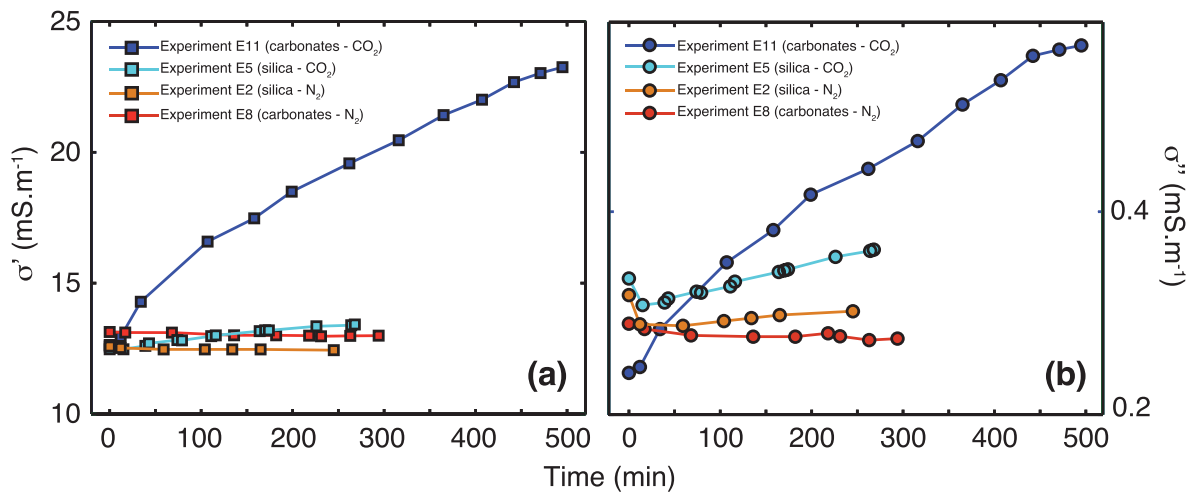

Figure 10. Evolution along time of the variations for the in-phase conductivity $\sigma^{\prime}$ and the quadrature conductivity $\sigma^{\prime \prime}$ measured at the frequency $f=12 \mathrm{kHz}$, during experiment E2, E5, E8 and E11. The initial conductivity of the water was $40 \mathrm{mS} \mathrm{m}^{-1}$.

(a)

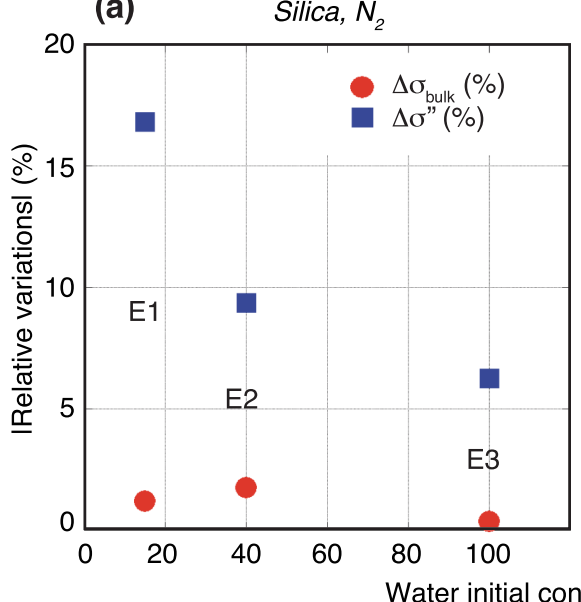

(b)

Carbonates, $\mathrm{CO}_{2}$

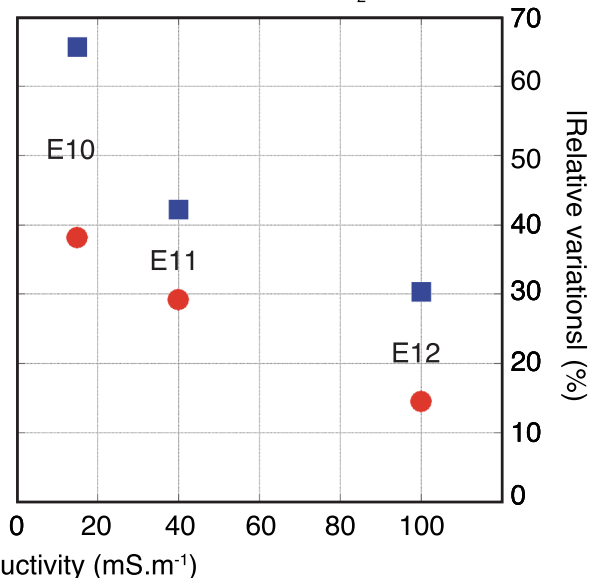

Figure 11. Comparison between the magnitude of the bulk conductivity relative variations and the quadrature conductivity relative variations observed when the main physicochemical phenomenon is desaturation (a: experiment E1, E2 and E3) or dissolution (b: experiment E10, E11 and E12). The quadrature conductivity is always more affected by gas injection than the bulk conductivity.

The absolute relative percentage variations are always greater for the quadrature conductivity than for the bulk conductivity. This point has been verified systematically for each of the 12 experiments, and this comparison is meaningful since both the bulk conductivity and the quadrature conductivity follows variations whose magnitude is way above the resolution limit of the measuring device $(1 \mathrm{mrad}$ for the measured phase and $0.1 \Omega$ for the measured resistance, see Radic 2004). This result suggests that the capacitive properties of the medium (in the intermediate-frequency range) are more strongly affected by gas injection than the ohmic properties.

\subsection{Origin of the intermediate frequency SIP response}

The results obtained during this series of experiment suggest that the intermediate frequency domain $\left(10^{2}-10^{5} \mathrm{~Hz}\right)$ is the frequency range for which the quadrature conductivity $\sigma^{\prime \prime}$ is the most sensitive to gas injection into saturated granular media. In this frequency range, it is generally accepted that the dominant polarization mechanism is the Maxwell-Wagner effect (e.g. Chen \& Or 2006a,b), which takes place at the interface between two materials of different electrical properties. In this study, two main processes have been distinguished. If the water saturation level decreases and that no dissolution processes occur then $\sigma^{\prime \prime}$ values decrease (e.g. injection of $\mathrm{N}_{2}$ ). If significant dissolution processes occur, they generate an increase of $\sigma^{\prime \prime}$ values. Those behaviours are in accordance with several studies showing the Maxwell-Wagner effect dependence with fluid conductivity and saturation level (e.g. de Lima \& Sharma 1992; Garrouch \& Sharma 1994; Leroy et al. 2008), which makes plausible the hypothesis of a significant Maxwell-Wagner contribution on the signals observed during this study. Other authors, such as Revil (2013), are in disagreement with the idea of an intermediatefrequency range dominated by the Maxwell-Wagner polarization and favour a low-frequency (Stern layer) polarization mechanism. However, even though there is not a total agreement on what is the dominant mechanism, all authors observe the same signal dependence with fluid conductivity and saturation level as in this study.

\subsubsection{Electronic environment}

The intermediate-frequency range is often disregarded in SIP studies, partly because many parasitic effects can be affecting the measured values: the influence of the electronic environment in the laboratory, the influence of the measuring device's own electronics, or the influence of the EM coupling between the measuring cables and the injection cables (e.g. Schmutz et al. 2014). In some cases, and particularly if the measured signal is weak, these external factors can affect significantly the measurement results.

In order to estimate the influence of such instrumental effects, we conducted a series of measurement on pure ohmic material (resistors), for which the measured phase values are supposed to be 
0 at all frequencies. We used resistors ranging from 30 to $660 \Omega$, so that we cover the same resistance range as during the experiments.

Details of the results are given in Appendix A.2. We found that non-zero phase values were observable, testifying to the existence of instrumental effects, and that their magnitude increases with frequency and with the resistance value. However, we showed that these instrumental effects cause variations always 1 or 2 orders of magnitude lower than the actual observed variations, indicating that the influence of instrumental effects is negligible, and therefore, that the observed variations result from physicochemical changes in the studied media (desaturation or dissolution processes). Therefore, in these experiments, the use of the SIP method, particularly in the intermediate-frequency range, produces supplementary information compared to what would have been retrieved from 'simple' resistivity monitoring.

\subsubsection{Electrode effects}

SIP measurements performed on water (Section 2.3) showed that non-zeros phase values were observed for frequencies above $100 \mathrm{~Hz}$, despite the non-polarizable nature of water. We have shown that the electronic environment of the experiment could not be considered as a significant contribution. Some authors suggest that this behaviour could be due to the electrode impedance contribution (Tirado et al. 2000). In recent studies, Huisman et al. (2014) observed that these effects become significant in the intermediate-frequency range $(f>1 \mathrm{kHz})$, which could be consistent with the data presented here, and Abdulsamad et al. (2014) indicate that the magnitude of these effects increases with contact impedance value.

The issue of electrode effects has not yet been much addressed quantitatively in published studies, but is likely to play a significant role in the behaviour observed in this experimental study, and should therefore be investigated more deeply in future work.

\section{CONCLUSIONS}

Through the different experiments performed in unconsolidated porous media, we have been able to distinguish the variations caused by changes of the effective water saturation level in the porous space from those resulting from the augmentation of dissolved ionic species in the saturating water, resulting from various dissolution processes.

This series of experiments therefore highlights the fact that the SIP technique is of significant interest for the monitoring and detection of $\mathrm{CO}_{2}$ transfers in a water-saturated unconsolidated granular media. In the intermediate-frequency range $\left(10^{2}-10^{5} \mathrm{~Hz}\right)$, the capacitive properties of the studied media are particularly affected by $\mathrm{CO}_{2}$ injection, with a larger magnitude than conductive properties. The SIP method could easily be used in the field instead of classical ERT surveys. Although the method is promising, work still needs to be done to better understand the origins of the SIP response in the intermediate-frequency range, and particularly to estimate the contribution of electrode effects.

\section{ACKNOWLEDGEMENTS}

We gratefully acknowledge the support of the $\mathrm{CO}_{2}$ research programme co-funded by ADEME, TOTAL and SCHLUMBERGER and their permission to publish this paper. However, the views expressed here are those of the authors who are solely responsible for any errors. We also address special thanks to A. Gendrin,
J. Desroches and M. Carney (SCHLUMBERGER) for their useful suggestions. Finally, we thank the editor and reviewers of the manuscript for their valuable comments. This is IPGP contribution no. 3773 .

\section{REFERENCES}

Abdulsamad, F., Florsch, N., Schmutz, M. \& Camerlynck, C., 2014. The paradox of the measuring electrodes in IP, in Proceedings of the $3 \mathrm{rd}$ International Workshop on Induced Polarization, pp. 48-49, eds Camerlynck, C., Chauris, H., Maineult, A. \& Schmutz, M., 6-9 April 2014, Oléron Island, France.

Auken, E., Doetsch, J., Fiandaca, G., Christiansen, A.V., Gazoty, A., Cahill, A.G. \& Jakobsen, R., 2014. Imaging subsurface migration of dissolved $\mathrm{CO}_{2}$ in a shallow aquifer using 3-D time-lapse electrical resistivity tomography, J. appl. Geophys., 101, 31-41.

Bergmann, P., Schmidt-Hattenberger, C., Kiessling, D., Rücker, C., Labitzke, T., Henninges, J., Baumann, G. \& Schütt, H., 2012. Surfacedownhole electrical resistivity tomography applied to monitoring of $\mathrm{CO}_{2}$ storage at Ketzin, Germany, Geophysics, 77, B253-B267.

Binley, A., 2015. R3t version 1.8, Software User Guide.

Binley, A. \& Kemna, A., 2005. DC resistivity and induced polarization methods, in Hydrogeophysics, pp. 129-156, eds Singh, V.P., Hubbard, S.S. \& Rubin, Y., Springer.

Binley, A., Slater, L.D., Fukes, M. \& Cassiani, G., 2005. Relationship between spectral induced polarization and hydraulic properties of saturated and unsaturated sandstone, Water Resour. Res., 41, W12417, doi:10.1029/2005WR004202.

Breede, K. \& Kemna, A., 2012. Spectral induced polarization measurements on variably saturated sand-clay mixtures, Near Surf. Geophys., 10, 479 489.

Breen, S.J., Carrigan, C.R., LaBrecque, D.J. \& Detwiler, R.L., 2012. Benchscale experiments to evaluate electrical resistivity tomography as a monitoring tool for geologic $\mathrm{CO}_{2}$ sequestration, Int. J. Greenhouse Gas Control, 9, 484-494.

Brewer, P.G., Chen, B., Warzinski, R., Baggeroer, A., Peltzer, E.T., Dunk, R.M. \& Walz, P., 2006. Three-dimensional acoustic monitoring and modeling of a deep-sea $\mathrm{CO}_{2}$ droplet cloud, Geophys. Res. Lett., 33, L23607, doi:10.1029/2006GL027181.

Carrigan, C.R., Yang, X., LaBrecque, D.J., Larsen, D., Freeman, D., Ramirez, A.L. \& Hovorka, S., 2013. Electrical resistance tomographic monitoring of $\mathrm{CO}_{2}$ movement in deep geologic reservoirs, Int. J. Greenhouse Gas Control, 18, 401-408.

Carroll, S., Hao, Y. \& Aines, R., 2009. Geochemical detection of carbon dioxide in dilute aquifers, Geochem. Trans., 10, doi:10.1186/1467-4866$10-4$.

Chelidze, T.L., Gueguen, Y. \& Ruffet, C., 1999. Electrical spectroscopy of porous rocks: a review-II. Experimental results and interpretation, Geophys. J. Int., 137, 16-34.

Chen, Y. \& Or, D., 2006a. Geometrical factors and interfacial processes affecting complex dielectric permittivity of partially saturated porous media, Water Resour. Res., 42, W06423, doi:10.1029/2005WR004744.

Chen, Y. \& Or, D., 2006b. Effects of Maxwell-Wagner polarization on soil complex dielectric permittivity under variable temperature and electrical conductivity, Water Resour. Res., 42, W06424, doi:10.1029/2005WR004590.

Clavaud, J.-B., 2001. Etude des propriétés de transport (hydraulique et électrique) des roches. Effets de la microstructure, de la présence de plusieurs fluides, de la fracturation et de l'interaction eau-roche, $P h D$ thesis, Université Paris-Diderot, Paris.

Cosenza, P., Ghorbani, A., Florsch, N. \& Revil, A., 2007. Effects of drying on the low-frequency electrical properties of Tournemire argillites, Pure appl. Geophys., 164, 2043-2066.

Dafflon, B., Wu, Y., Hubbard, S.S., Birkholzer, J.T., Daley, T.M., Pugh, J.D. \& Trautz, R.C., 2013. Monitoring $\mathrm{CO}_{2}$ intrusion and associated geochemical transformations in a shallow groundwater system using complex electrical methods, Environ. Sci. Technol., 47, 314-321. 
de Lima, O.A. \& Sharma, M.M., 1992. A generalized Maxwell-Wagner theory for membrane polarization in shaly sands, Geophysics, 57, 431440

Edwards, N. \& Riggs, J., 2003. Automated monitoring of soil respiration: a moving chamber design, Soil Sci. Soc. Am. J., 67, 1266-1271.

Garrouch, A.A. \& Sharma, M.M., 1994. The influence of clay content, salinity, stress, and wettability on the dielectric properties of brine-saturated rocks: $10 \mathrm{~Hz}$ to $10 \mathrm{MHz}$, Geophysics, 59, 909-917.

Hallof, P.G., 1974. The IP phase measurement and inductive coupling, Geophysics, 39, 650-665.

Huisman, J.A., Vereecken, H., Zimmermann, E., Treichel, A. \& Haegel, F.H., 2014. Evaluation of a correction procedure to remove electrode contact impedance effects from broadband SIP measurements, in Proceedings of the 3rd International Workshop on Induced Polarization, pp. 56-57, eds Camerlynck, C., Chauris, H., Maineult, A. \& Schmutz, M., 6-9 April 2014, Oléron Island, France.

Ishai, P.B., Talary, M.S., Caduff, A., Levy, E. \& Feldman, Y., 2013. Electrode polarization in dielectric measurements: a review, Meas. Sci. Technol., 24, 102001, doi:10.1088/0957-0233/24/10/102001.

Jougnot, D., Ghorbani, A., Revil, A., Leroy, P. \& Cosenza, P., 2010. Spectral induced polarization of partially saturated clay-rocks: a mechanistic approach, Geophys. J. Int., 180, 210-224.

Kemna, A., Binley, A. \& Slater, L., 2004. Crosshole IP imaging for engineering and environmental applications, Geophysics, 69, 97-107.

Kiessling, D., Schmidt-Hattenberger, C., Schuett, H., Schilling, F., Krueger, K., Schoebel, B. \& COSINK Group, 2010. Geoelectrical methods for monitoring geological $\mathrm{CO}_{2}$ storage: first results from cross-hole and surface-downhole measurements from the $\mathrm{CO}_{2}$ SINK test site at Ketzin (Germany), Int. J. Greenhouse Gas Control, 4, 816-826.

Kremer, T., Schmutz, M., Leroy, P., Agrinier, P. \& Maineult, A., 2016. Modeling the spectral induced polarization response of water saturated sands in the intermediate frequency range $\left(10^{2}-10^{5} \mathrm{~Hz}\right)$ using mechanistic and empirical approaches, Geophys. J. Int., in press, doi:10.1093/gji/ggw334.

Leroy, P., Revil, A., Kemna, A., Cosenza, P. \& Ghorbani, A., 2008. Complex conductivity of water-saturated packs of glass beads, J. Colloid Interface Sci., 321, 103-117.

Lesmes, D.P. \& Frye, K.M., 2001. Influence of pore fluid chemistry on the complex conductivity and induced polarization responses of Berea sandstone, J. geophys. Res., 106(B3), 4079-4090.

Liu, G., 2012. Carbon dioxide geological storage: monitoring technologies review, in Greenhouse Gases-Capturing, Utilization and Reduction, pp. 299-338, ed. Liu, G., InTech.

Madsen, R., Xu, L., Claassen, B. \& McDermitt, D., 2009. Surface monitoring method for carbon capture and storage projects, Energy Proc., 1, 21612168.

Marshall, D. \& Madden, T., 1959. Electrode and membrane polarization, M.I.T. Report to A.E.C., RME 3157.

Nakatsuka, Y., Xue, Z., Garcia, H. \& Matsuoka, T., 2010. Experimental study on $\mathrm{CO}_{2}$ monitoring and quantification of stored $\mathrm{CO}_{2}$ in saline formations using resistivity measurements, Int. J. Greenhouse Gas Control, 4, 209-216.

Pelton, W., 1977. Interpretation of complex resistivity and dielectric data, PhD thesis, University of Utah.

Peter, A., Lamert, H., Beyer, M., Hornbruch, G., Heinrich, B., Schulz, A. \& Dahmke, A., 2012. Investigation of the geochemical impact of $\mathrm{CO}_{2}$ on shallow groundwater: design and implementation of a $\mathrm{CO}_{2}$ injection test in Northeast Germany, Environ. Earth Sci., 67, 335-349.

Radic, T., 2004. Elimination of cable effects while multi-channel SIP measurements, in Near Surface 2004 - 10th European Meeting of Environmental and Engineering Geophysics, Utrecht, The Netherlands.

Revil, A., 2013. Effective conductivity and permittivity of unsaturated porous materials in the frequency range $1 \mathrm{mHz}-1 \mathrm{GHz}$, Water Resour. Res., 49, 306-327.

Revil, A. \& Florsch, N., 2010. Determination of permeability from spectral induced polarization in granular media, Geophys. J. Int., 181, 1480-1498.

Revil, A. \& Skold, M., 2011. Salinity dependence of spectral induced polarization in sands and sandstones, Geophys. J. Int., 187, 813-824.
Schmutz, M., Ghorbani, A., Vaudelet, P. \& Blondel, A., 2014. Cable arrangement to reduce electromagnetic coupling effects in spectral-induced polarization studies, Geophysics, 79, A1-A5.

Schmutz, M., Ghorbani, A., Vaudelet, P. \& Revil, A., 2011. Spectral induced polarization detects cracks and distinguishes between open- and clayfilled fractures, J. Environ. Eng. Geophys., 16, 85-91.

Schwan, H.P., 1968. Electrode polarization impedance and measurements in biological materials, Ann. N.Y. Acad. Sci., 148, 191-209.

Scott, J.B. \& Barker, R.D., 2003. Determining pore-throat size in PermoTriassic sandstones from low-frequency electrical spectroscopy, Geophys. Res. Lett., 30, 1450, doi:10.1029/2003GL016951.

Skold, M., Revil, A. \& Vaudelet, P., 2011. The pH dependence of spectral induced polarization of silica sands: experiment and modeling, Geophys. Res. Lett., 38, L12304, doi:10.1029/2011GL047748.

Tirado, M.C., Arroyo, F.J., Delgado, A.V. \& Grosse, C., 2000. Measurement of the low-frequency dielectric properties of colloidal suspensions: comparison between different methods, J. Colloid Interface Sci., 227, 141-146.

Ulrich, C. \& Slater, L., 2004. Induced polarization measurements on unsaturated, unconsolidated sands, Geophysics, 69, 762-771.

Vaudelet, P., Revil, A., Schmutz, M., Franceschi, M. \& Bégassat, P., 2011. Induced polarization signatures of cations exhibiting differential sorption behaviors in saturated sands, Water Resour. Res., 47, W02526, doi:10.1029/2010WR009310.

Vialle, S., Contraires, S., Zinzsner, B., Clavaud, J.-B., Mahiouz, K., Zuddas, P. \& Zamora, M., 2014. Percolation of $\mathrm{CO}_{2}$-rich fluids in a limestone sample: evolution of hydraulic, electrical, chemical, and structural properties, J. geophys. Res., 119, 2828-2847.

Weller, A., Slater, L., Nordsiek, S. \& Ntarlagiannis, D., 2010. On the estimation of specific surface per unit pore volume from induced polarization: a robust empirical relation fits multiple data sets, Geophysics, 75, WA105WA112.

Weller, A., Breede, K., Slater, L. \& Nordsiek, S., 2011. Effect of changing water salinity on complex conductivity spectra of sandstones, Geophysics, 76, F315-F327.

Xue, Z., Tanase, D. \& Watanabe, J., 2006. Estimation of $\mathrm{CO}_{2}$ saturation from time-lapse $\mathrm{CO}_{2}$ well logging in an onshore aquifer, Nagaoka, Japan, Explor. Geophys., 37, 19-29.

Zonge, K.L. \& Hughes, L.J., 1985. Effect of electrode contact resistance on electric field measurements, in SEG Technical Program Expanded Abstracts, SEG-1985-0231, Society of Exploration Geophysicists.

Zonge, K.L. \& Wynn, J.C., 1975. Recent advances and applications in complex resistivity measurements, Geophysics, 40, 851-864.

\section{APPENDIX A.1 - NUMERICAL SENSITIVITY STUDY}

It was conducted as follows. We numerically generated a homogeneous synthetic resistivity distribution of $25 \Omega \mathrm{m}$ that has the same geometry as our cylindrical reservoir. Using the forward modeling software R3t, we simulated a resistance measurement using a given electrode configuration. We repeated the process on the same resistivity distribution in which we included a high resistivity $(100 \Omega \mathrm{m})$ cylinder (Fig. Ala). From the two resistance values obtained, we calculated the resistance increase percentage $\delta R_{\%}$ generated by the inclusion. Calculating $\delta R_{\%}$ for several lateral positions of the cylindrical inclusion leads to an evaluation of the sensitivity spatial distribution for a given electrode configuration (Fig. A1b). These processes were applied to all the possible electrode configurations for a cylinder instrumented with six electrodes displayed on the same horizontal plan, each of them forming a $60^{\circ}$ angle with their closest neighbour and the central axis of the cylinder. Among all the configurations tested, the so-called ' $120^{\circ}$ configuration' proved to show the best compromise between high sensitivity values and homogeneity of the sensitivity spatial distribution over a horizontal 
(a)

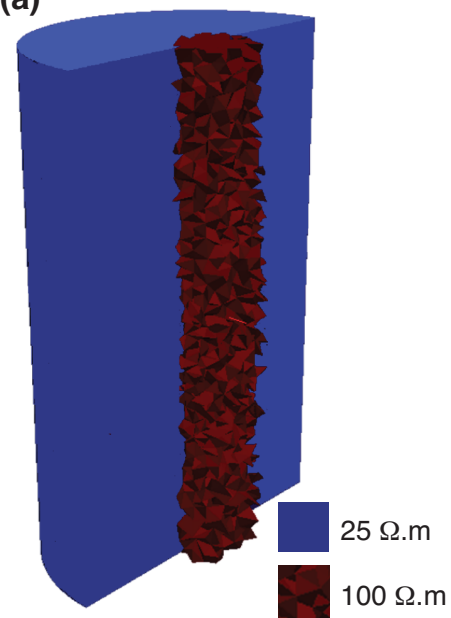

(b)

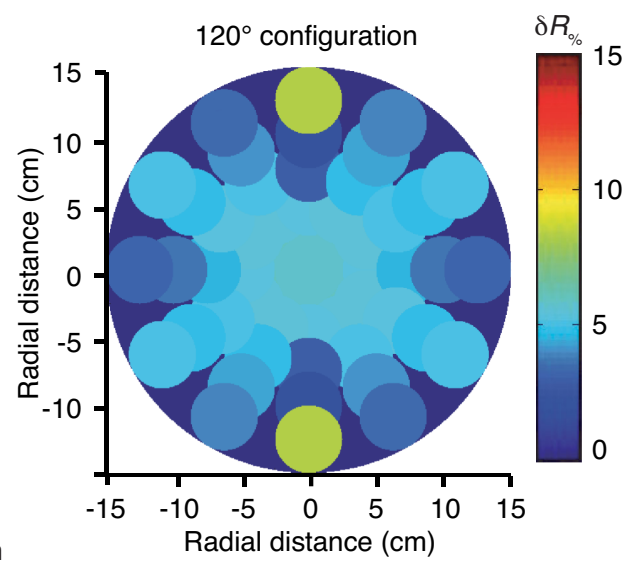

Figure A1. Evaluation of the sensitivity distribution of a given electrode configuration. (a) Inclusion of a $100 \Omega$ m cylindrical inclusion embedded in a $25 \Omega \mathrm{m}$ homogeneous cylinder. (b) 2 -D sensitivity distribution of the ' $120^{\circ}$ configuration', that was chosen to perform the experiments.

section of the cylinder (Fig. A1b). The homogeneity constraint was particularly important since we had no certainty about what would be the spatial repartition of the $\mathrm{CO}_{2}$ that was injected.

\section{APPENDIX A.2 - MEASUREMENTS ON RESISTORS}

Fig. A2(a) shows the phase spectra measured in the frequency range $0.5 \mathrm{~Hz}-20 \mathrm{kHz}$, on five resistors of different values, going from 30 to $660 \Omega$, which corresponds to the resistivity range observed during the different experiments.

It reveals that the phase spectrum is not flat when approaching the intermediate-frequency range. Small phase values $(<1 \mathrm{mrad})$ are measured, indicating that some instrumental effects are disturbing the measurements. Even though the exact nature of these instrumental effects is not determined, their magnitude seems to be correlated with the resistor value (see Fig. A2b). This correlation means that during the experiments, resistivity variations can cause a change in phase measurement (and hence on $\sigma^{\prime \prime}$ values) that originate from instrumental effects, and not from a physical modification of the medium. It is therefore necessary to check whether this instrumental influence is significant regarding the magnitude of the variations observed during the experiments.

At the frequency $f=12 \mathrm{kHz}$, a simple power law can fit reasonably the data (adjustment coefficient $r^{2}=0.997$ ). Using this power law, we estimated the influence of resistivity variations on the measured phase. We concluded that for all processes (desaturation drop or dissolution increase), the instrumental effects generated variations always 1 or 2 order of magnitude lower than the actual observed variations, indicating that the influence of instrumental effects was negligible, and therefore, that the observed variations resulted from physicochemical changes in the studied medium, that is, desaturation or dissolution processes.
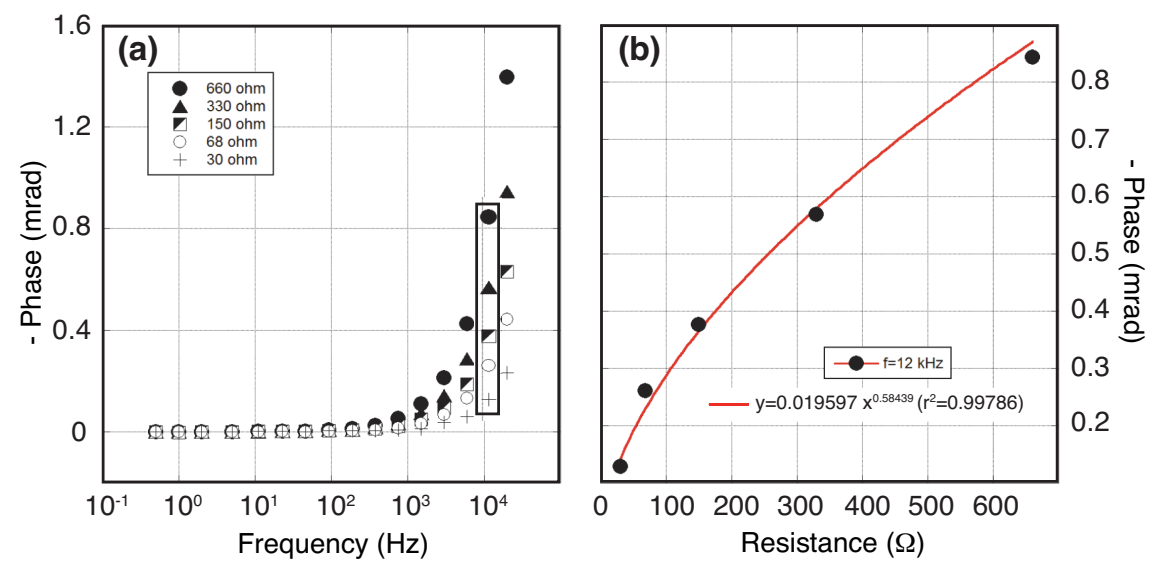

Figure A2. (a) Phase spectra measured on ohmic resistors for five different values in the frequency range $0.5 \mathrm{~Hz}-20 \mathrm{kHz}$. Measured values tend to increase when approaching the intermediate-frequency range, and increase with the resistance value. (b) Representation of the dependence of the measured phase value with the resistance value, for the specific frequency $12 \mathrm{kHz}$. The evolution can be fitted with a simple power law. 